\title{
Observed Evolution of a California Undercurrent Eddy
}

\author{
Jacob M. Steinberg, Noel A. Pelland, And Charles C. Eriksen \\ School of Oceanography, University of Washington, Seattle, Washington
}

(Manuscript received 23 February 2018, in final form 12 November 2018)

\begin{abstract}
A California Undercurrent eddy (Cuddy) was repeatedly surveyed using multiple Seagliders for over three months. Found and tracked off of the Washington-Vancouver Island coasts, this Cuddy traveled over $400 \mathrm{~km}$, remaining between the 1000- and 2000-m isobaths, as it was swept along in poleward flow of the California Current System. Three Seagliders made repeat bisecting transects of the Cuddy core capturing its detailed three-dimensional structure in time. Its evolution was analyzed through comparison of 11 independent Cuddy "snapshots." A two dimensional Gaussian model fit to the geopotential anomaly field for each snapshot allowed computation of dynamic fields inaccessible in Seaglider profiles alone. Results indicate that the Cuddy decayed as its core waters became less isolated over time: Cuddy total mechanical energy (kinetic + potential), salt content, and the magnitude of the core potential vorticity anomaly decreased. Core spice and dissolved oxygen variance increased tenfold, and thermohaline fine structure, suggestive of lateral intrusions, was observed progressively closer to the eddy core. The estimated gradientwind balanced velocity field similarly weakened as the Rossby number decreased to 0.32 from an initial value of 0.48 . The observed changes in eddy properties occurred as the Cuddy was exposed to changes in the background stratification and Coriolis parameter as it translated alongshore. Idealized modeling of eddy adjustment indicates that both erosion and changing background conditions are required to explain the observed eddy changes. Adjustment in response to both effects simultaneously leads to changes in eddy properties qualitatively consistent with those observed.
\end{abstract}

\section{Introduction}

Eddies play important yet often undetected roles in the transport and distribution of oceanic heat, salt, and nutrients. They occupy a range of spatial scales, are found at various depths in all oceans, and are inherently transient. Submesoscale coherent vortices (SCVs) — gradientwind balanced, preferentially anticyclonic, subsurface intensified, isolated lenses with horizontal length scales comparable to or smaller than the first baroclinic deformation radius and Rossby numbers $\mathscr{O}(1)$ (McWilliams 1985; D'Asaro 1988b) - are a distinctive class of longlived eddies capable of trapping and transporting core waters for many months over hundreds of kilometers (McWilliams 2016). Their ubiquity, potential role in the transfer of energy from large to small scales, and ability to redistribute bulk quantities of heat, salt, and nutrients motivates the study of their behavior and evolution.

The prevalence of SCVs has been recognized through frequent observations that are often unintentional (e.g.,

Corresponding author: Jacob Steinberg, jms000@uw.edu
Huyer et al. 1998; Collins et al. 2013). Due to a strong potential vorticity gradient bounding an isolated relatively homogeneous core (Elliott and Sanford 1986), SCVs that avoid disintegration due to collisions with topography (e.g., Torres and Gomez-Valdes 2017), or merging and interaction with other eddies, exhibit a relatively slow decay driven by dissipative processes. Persistent, high-resolution observations of a Mediterranean water eddy ("Meddy," an archetypal SCV) over a multiyear period illustrated losses of heat and salt driven by lateral intrusions (Armi et al. 1989; Hebert et al. 1990), similar to those observed elsewhere along sharp thermohaline fronts (e.g., Shcherbina et al. 2009, 2010). Similar extended tracking and observation of individual features is informative of the processes moderating SCV translation, longevity, and decay, but is a challenge due to these eddies' small size and subsurface intensification and is as a consequence more rare. As a result, quantitative estimates of the contribution of coherent submesoscale eddies to regional and global tracer transport and energy flux remain poorly constrained. Improvements in high-resolution models have allowed exploration of 
SCV propagation and evolution behavior (e.g., McWilliams et al. 1986; McWilliams and Gent 1986; McWilliams 1988; Morel and McWilliams 1997; Molemaker et al. 2015), though further observational counterparts are needed (McWilliams 2016). In this article, we describe results from persistent, three-dimensional spatial surveys of a submesoscale California Current eddy using nearcontinuous autonomous observations over three months.

California Undercurrent (CU) eddies, termed "Cuddies" (Garfield et al. 1999; Collins et al. 2013; Pelland et al. 2013), have characteristics similar to those of SCVs and are frequently found in the California Current System (CCS) in the eastern North Pacific Ocean. Cuddies are long-lived monopole anticyclones, with radii $10-30 \mathrm{~km}$ and velocity maxima at the base of the permanent pycnocline. Cuddies originate along the continental slope in the $\mathrm{CU}$, which is a wind-forced subsurface jet driven by local and remote alongshore wind forcing, carrying warm, saline, low oxygen, high nutrient Pacific Equatorial Waters (PEW) to higher latitudes (Hickey 1979; Huyer et al. 1998; Chereskin et al. 2000; Connolly et al. 2014; Thomson and Krassovski 2015). Peak poleward velocities of $0.3-0.5 \mathrm{~m} \mathrm{~s}^{-1}$ are observed in late summer and early autumn offshore of the continental shelf break and at a core depth between $100-300 \mathrm{~m}$; in the northern CCS, the peak PEW signature is found on the potential density surface $\rho_{\theta}=1026.55 \mathrm{~kg} \mathrm{~m}^{-3}$ (Hickey 1979; Thomson and Krassovski 2010; Pelland et al. 2013). Offshore of the core jet, the broader surface-intensified California Current (CC) flows equatorward carrying waters from the North Pacific (Auad et al. 2011). In winter, north of $43^{\circ} \mathrm{N}$ and near the shelf break, surface currents become poleward and are denoted the Davidson Current (Hickey 1979).

Collins et al. (2013) analyzed looping anticyclonic RAFOS float trajectories to track Cuddies originating along the continental slope within the CCS. Floats became trapped in Cuddies as they formed and translated offshore, revealing stable, west-southwestward drift into the more quiescent North Pacific interior. This is consistent with expectations of eddy self-advection due to the $\beta$ effect (McWilliams et al. 1986; McWilliams and Gent 1986; Morel and McWilliams 1997), observed for surfaceintensified mesoscale eddies in a global eddy census using gridded altimetry (Chelton et al. 2011). A notable Cuddy trajectory ended $1600 \mathrm{~km}$ to the southwest of the initiation of looping, providing further evidence of these eddies' potentially long life (Collins et al. 2013). Lukas and Santiago-Mandujano (2001) corroborate these results with observations of an extreme water mass anomaly near the Hawaiian Islands, which they conclude likely originated in the CU. The properties of mature eddies are consistent with a range of generation scenarios including baroclinic instability, topographic interaction, and bottom friction effects. High-resolution models explain Cuddy formation as resulting from a combination of flow separation over complex topography and frictional interactions with the continental slope (Molemaker et al. 2015). Torres and Gomez-Valdes (2017) suggest that the meandering of the $\mathrm{CU}$ induced by synoptic-scale wind events can also play an important role. Observations capturing the formation of a Peru-Chile Undercurrent eddy, a type similar to Cuddies, corroborate flow separation over rough topography and bottom friction as a formation mechanism (Thomsen et al. 2016). These observations affirm eddy formation as a complex process and highlight the importance of frictional bottom interactions suggested by D'Asaro (1988a) and Molemaker et al. (2015).

Cuddies contain core water properties similar to those of the $\mathrm{CU}$, which distinguish them from cooler and fresher background waters as they move offshore. Spice, a state variable defined as a measure of thermohaline variability along isopycnals (Flament 2002) is useful for identifying $\mathrm{CU}$ water mass properties within Cuddies as well as interleaving of water masses as they move into cooler fresher offshore waters. This pathway for the offshore advection of PEW water can help explain the observed poleward weakening of the $\mathrm{CU}$ water mass signal as it mixes with the surrounding Pacific Subarctic Upper Water (Thomson and Krassovski 2010). Observations of twenty individual Cuddies in a sequence of repeat Seaglider sections carried out over the Washington continental slope between 2003 and 2009 are consistent with the hypothesis that these eddies act as a primary agent of lateral stirring between the CU and its surroundings (Pelland et al. 2013). By estimating eddy bulk properties, frequency of occurrence, and the average decay of heat and salt content in the CU with latitude, Pelland et al. (2013) estimated that $44 \%$ of the heat and salt lost from the $\mathrm{CU}$ as it flows poleward in this region may move offshore via Cuddies. These observations also revealed instances of enhanced fine structure at Cuddy edges, suggestive of lateral intrusions, though the role that intrusions play in the decay of typical Cuddies remains unclear (Pelland et al. 2013).

To investigate mechanisms moderating eddy evolution, we deployed multiple Seaglider autonomous underwater vehicles (AUVs) to identify and track a single Cuddy in the same region sampled by Pelland et al. (2013), with the intent of continuously observing the structure and decay of an individual Cuddy for as long as vehicle endurance and eddy detectability allowed. These surveys combine analysis methods developed from repeat observations of other types of SCVs (D'Asaro 1988b; Elliott and Sanford 1986; Hebert et al. 1990) with AUV technology that has the ability to provide relatively 


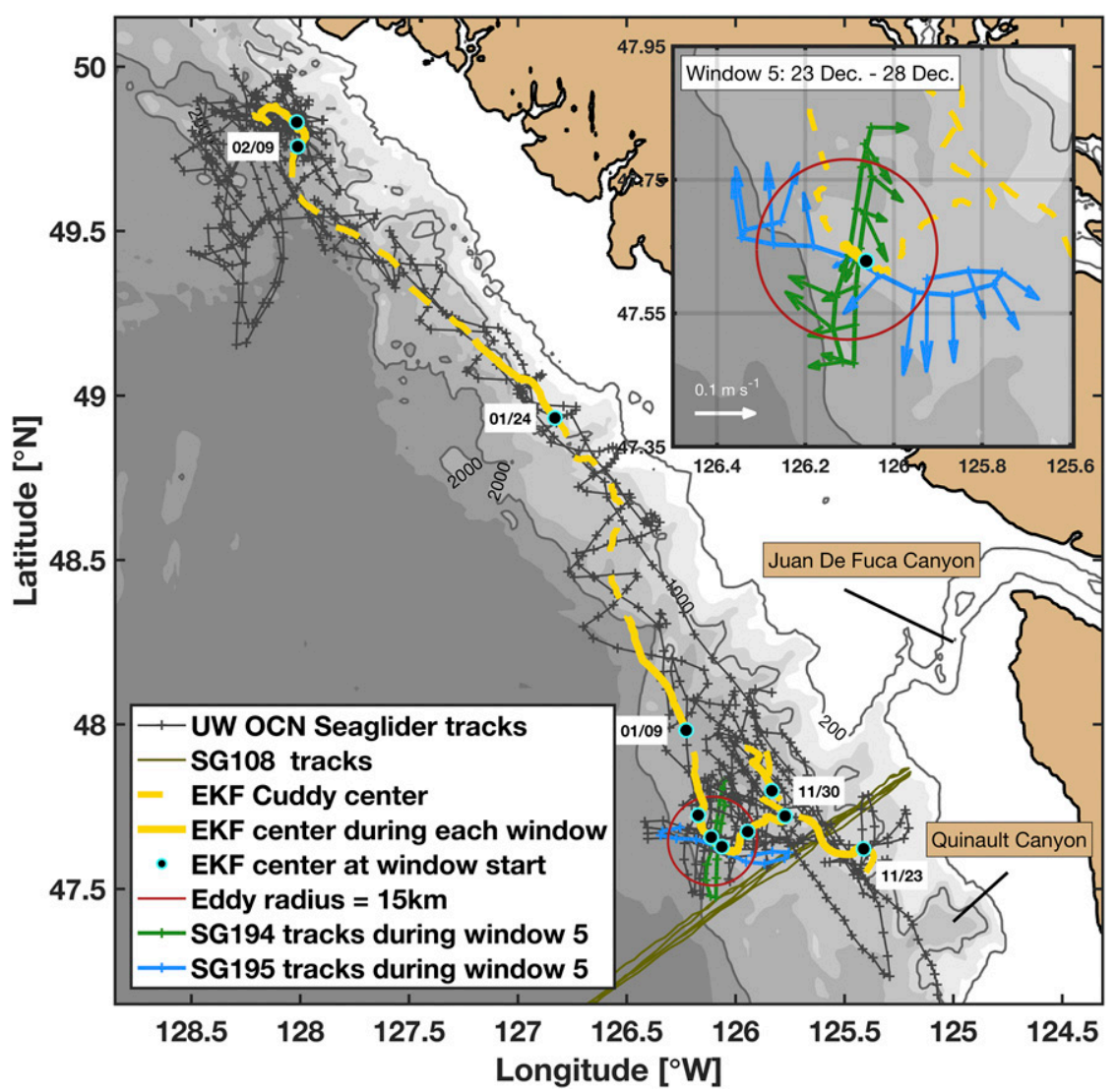

FIG. 1. SG189, SG194, and SG195 tracks from 20 Nov 2013 to 21 Mar 2014. Solid and dashed yellow lines are the Cuddy center estimate. Solid portions detail the Cuddy translation path during discrete time windows (Fig. 3e). Scattered in black with blue trim are Cuddy center positions at the start of each time window (select start times labeled). Glider paths and dive-cycle midpoints are in gray with SG108 cross-shore transects completed between 20 Nov and 20 Jan in dark green. The red circle is the estimated Cuddy radius at the fifth example time window. Gray shading indicates depth levels with the 200-, 1000-, and 2000-m contours added. The inset provides detail of a single time window with the tracks of two gliders (194 in green and 195 in blue) and DAC vectors for each dive-climb cycle.

high-resolution spatial and temporal observations over weeks or months (Martin et al. 2009; Bosse et al. 2016). The main goals of this study were to dynamically describe a Cuddy's structure, observe its evolution, and quantify its decay. The following sections detail the methods of data collection and analysis, along with the eddy's kinematic and dynamic evolution, core property changes, and observations of thermohaline interleaving. A discussion and conclusions follow, including consideration of idealized eddy adjustment to the surrounding environment and partial erosion.

\section{Data collection}

In October 2013, three Seagliders ("gliders"), designated SG189, SG194, and SG195 (Eriksen 2017a,b,c), were deployed from a chartered sport fishing vessel from Wesport, Washington, to find and continuously survey a single Cuddy. An additional glider, SG108, operated by the University of Washington Applied Physics Laboratory as part of the Northwest Association of Networked Ocean Observing Systems (www.nanoos.org), simultaneously carried out repeat transects along a track crossing the Washington continental slope (Fig. 1). This glider provided a simultaneous description of the background state.

Seagliders are buoyancy driven, autonomous underwater vehicles that profile the upper $1000 \mathrm{~m}$ of the water column measuring temperature, conductivity, and pressure on both ascent and descent (Eriksen et al. 2001). The result of each $\sim 8 \mathrm{~h}$ glider dive-climb cycle is pair of vertical profiles along a sawtooth (nominal 1:3 vertical:horizontal 
glide slope) path roughly $6 \mathrm{~km}$ in horizontal length. Gliders in this study sampled temperature and conductivity using a SeaBird Electronics thermistor and conductivity cell similar to those employed on SeaBird's SBE-3 and SBE-4 instruments, which were processed to estimate salinity $S$ using methods consistent with those described in Pelland et al. (2013, 2016). These measurements allowed for computation of potential temperature $\theta\left({ }^{\circ} \mathrm{C}\right)$ referenced to the sea surface, potential density $\sigma_{\theta}\left(=\rho_{\theta}-1000 \mathrm{~kg} \mathrm{~m}^{-3}\right)$, and spice $\pi_{\theta}$. Spice is used to highlight different water mass properties and to identify Cuddy and CU waters. For a given density, high spice is associated with warm salty Cuddy core water. Seagliders also estimate a surface to $1000 \mathrm{~m}$ depth-averaged current (DAC), with $0.01 \mathrm{~m} \mathrm{~s}^{-1}$ accuracy, over each dive-climb cycle from the difference between GPS-tracked overground and dead-reckoned displacements. Dissolved oxygen concentration per unit mass $\mathrm{O}_{2}$ (units of $\mu \mathrm{mol} \mathrm{kg}{ }^{-1}$ ) was also measured using the Aanderaa 4330 Optode, in addition to optical backscatter on $470 \mathrm{~nm}$ and $700 \mathrm{~nm}$ wavelengths and chlorophyll fluorescence using the WETLabs ECO Puck.

An individual Cuddy was found on 20 November 2013 and tracked along the continental slope offshore of Washington and Vancouver Island coasts (Fig. 1). Tracking commenced at $47.55^{\circ} \mathrm{N}, 125.4^{\circ} \mathrm{W}$ and ended on approximately 25 February 2014 at $49.8^{\circ} \mathrm{N}, 128.1^{\circ} \mathrm{W}$ due to the inability to further identify a coherent Cuddy from the data returned by the gliders. This region falls within the northern portion of the CCS where winter flow at the shelf break is on average poleward both at the surface and at depth (Pelland et al. 2013). In this study, the Cuddy path was largely poleward, suggesting that this eddy was caught in the prevailing background flows before reaching the quiescent interior North $\mathrm{Pa}$ cific Ocean. Glider tracks completed during the Cuddy tracking period, as well as an estimated eddy translation path, highlight this poleward propagation (Fig. 1). Eddy center position estimates are separated into segments contributing to Cuddy evolution analysis described below. Including SG108, a total of 1201 glider dive-climb cycles were carried out between 20 November 2013 and 21 March 2014 of which 972 profiles contributed in constructing Cuddy snapshots.

During tracking, basic eddy properties and background flow were estimated by assimilating glider observations of isopycnal vertical displacement and depth-averaged current into a simplified model using the extended Kalman filter (EKF). The EKF is a useful tool for sequentially estimating the properties of systems such as eddies, in which observations are nonlinear functions of the system state (Ide and Ghil 1998a,b). After completion of the surveys,

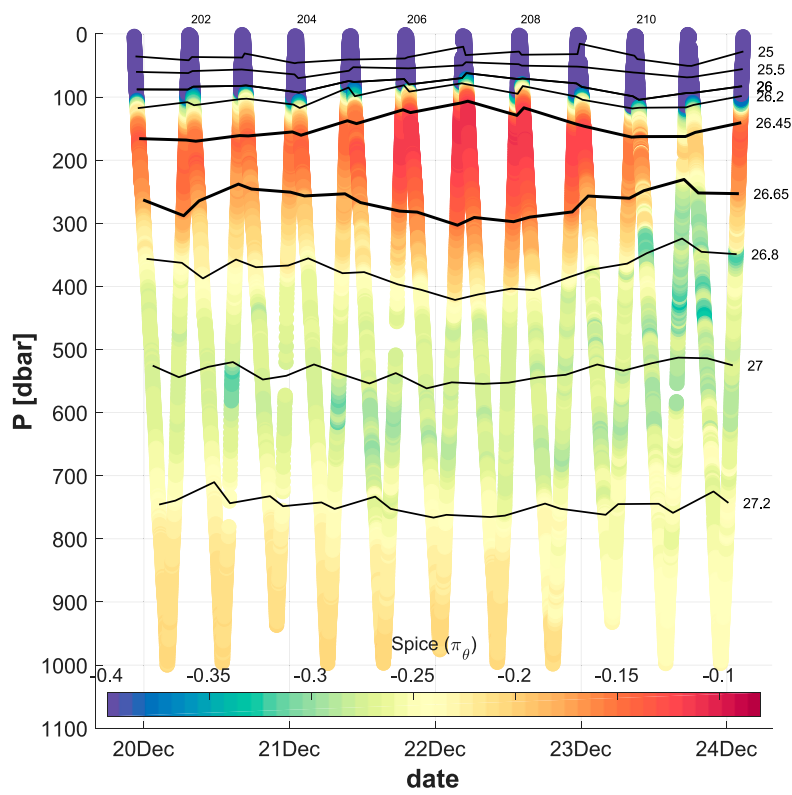

FIG. 2. A set of 11 dive-climb cycles (in pressure and time) collected by SG195 during an example crossing of the target Cuddy. Even glider dive-climb cycles are numbered. Color indicates spice (section 2); the deep red lens at 200-m depth is the Cuddy core. Isopycnals are contoured in black, and deflection bounding the core lens highlights the Cuddy's vertical extent.

a "smoother" - an algorithm for refining initial EKF estimates based on data collected at later times (Gelb 1974) — was applied, and estimates of eddy position, background flow, and position uncertainty derived from this smoother are used in the analyses that follow. The simple eddy model and estimation procedure are summarized in appendix A. Pelland et al. (2018) describes the EKF and smoother methodology and its application to automated glider navigation in greater detail.

\section{Methods}

\section{a. Cuddy tracking}

Throughout the tracking period, the survey gliders were oriented and directed to make repeat transects across the Cuddy. A complete transect was composed typically of eight dive-climb cycles taking 2-3 days to complete. A total of 98 Cuddy transects of lengths between 6 and $53 \mathrm{~km}$ were collected. Of these, 58 transects of at least $25 \mathrm{~km}$ are included in this analysis. Nominally, one glider was oriented north-south while a second was oriented eastwest; northwest-southeast or northeast-southwest tracks (Fig. 1) are due to the east-west transects taking place relative to a poleward-propagating eddy center. 


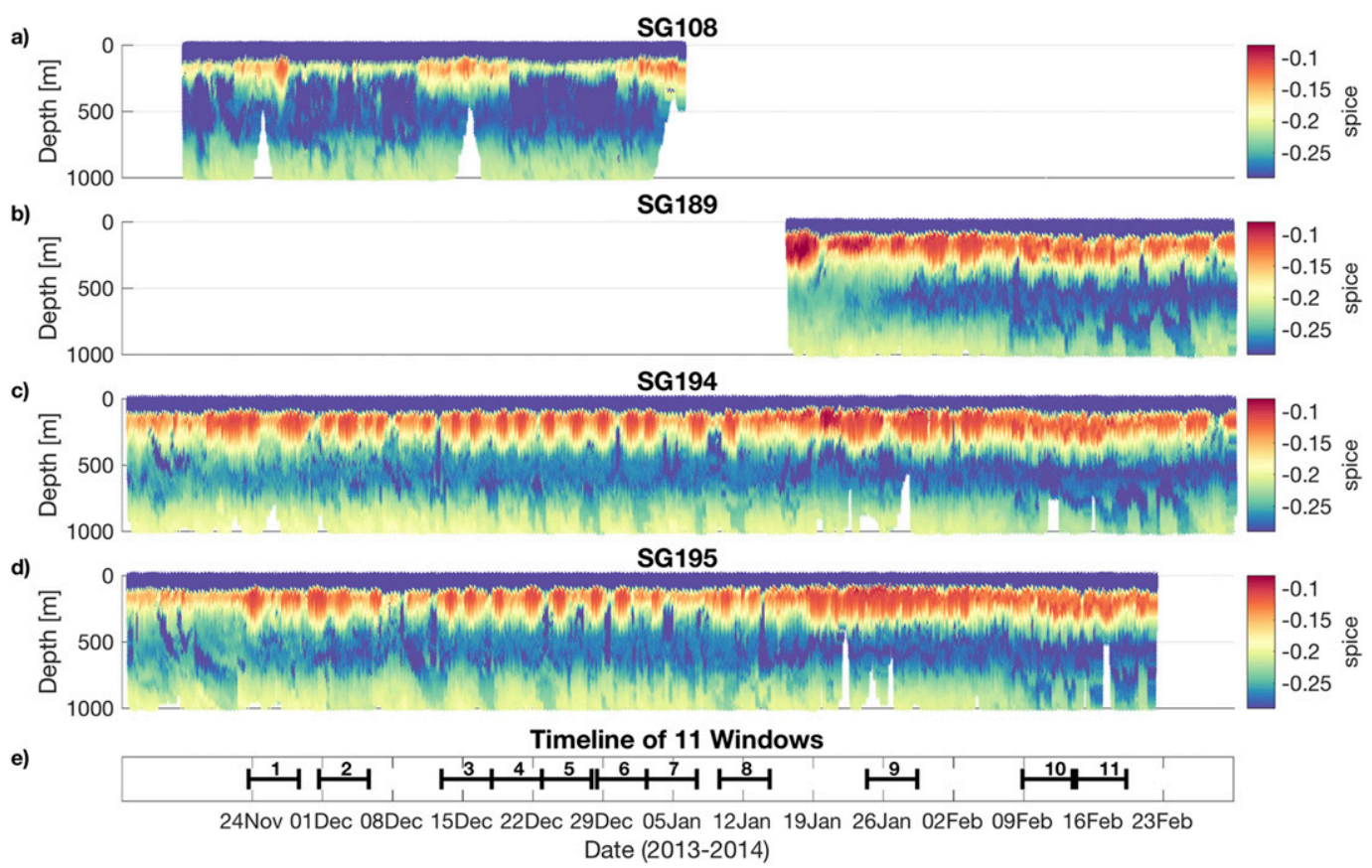

FIG. 3. (a)-(d) Spice (section 2) collected by all gliders in this study vs time and depth. For dedicated survey gliders (other than SG108), instances of high spice in the main pycnocline indicate the center of a Cuddy transect (e.g., Fig. 2). The periodic high spice sections made by SG108 instead reflect its movement on and offshore into and out of CU waters. (e) Timeline of the period spanned by each analysis window. Gaps in this timeline in early December and mid-January reflect times during which Cuddy translation speeds were increased and tracking became more difficult.

Profile data from a sample transect (11 dive-climb cycles collected by SG195 in December 2013; Fig. 2) illustrate the relatively warm, saline weakly stratified layer at the core of the target Cuddy. Here, stratification refers to the magnitude of the vertical derivative of potential density.

The ability to effectively survey the target Cuddy varied through time due to changes in glider availability and communications, details in piloting strategy, and eddy translation speed. Translation speeds approached $0.1 \mathrm{~m} \mathrm{~s}^{-1}$ at times, with a net speed of $0.084 \mathrm{~m} \mathrm{~s}^{-1}$ poleward along the continental slope during the period 6 January-10 February 2014, though with considerably lower speeds previous and subsequent to this 5 -week interval of fast translation. Survey tracks were coarser during the period of rapid poleward translation, but still sufficient to provide bulk estimates of eddy structure at some times.

\section{b. Processing framework}

Throughout the survey period and for the dedicated survey gliders, instances of elevated spice in the main pycnocline (200-m depth), and greater vertical extent of high spice waters, indicate the center of a cross-eddy transect similar to that in Fig. 2 (Fig. 3). Recall that
SG108 (Fig. 3a) did not sample the Cuddy repeatedly; the variability in spice over longer periods than the other gliders reflects its sampling of CU waters near the shelf break, then cooler, fresher offshore waters on successive cross-slope transects.

All profiles were referenced to the moving Cuddy center position in space and time, using the EKF smoother eddy center estimates (appendix A). The position of the center at the time of each glider sample was determined and radial distance was computed assuming linear Cuddy translation between each EKF estimate. Each profile was vertically bin averaged using overlapping bins to grid each profile in $2-\mathrm{m}$ increments from 0 to $150 \mathrm{~m}, 5-\mathrm{m}$ increments from 150 to $300 \mathrm{~m}$, and $10-\mathrm{m}$ increments deeper than $300 \mathrm{~m}$; bin increments were chosen to reflect varying glider temporal sampling rates. Profiles without bin averaging were also considered for analysis of vertical fine structure as will be discussed below.

A total of 11 nonoverlapping time windows, each 5 days long, were used to create 11 Cuddy "snapshots" that could be compared with one another. Contributions to each snapshot from gliders varied throughout the survey period, with at least two profiling the Cuddy at a given time (Fig. 3). The time bounds of each window 
were chosen to reflect the availability of high-quality transects and to maximize the spatial extent of available Cuddy measurements.

In adopting this procedure, it is assumed that changes in Cuddy vertical-radial structure over the course of each 5-day window are small relative to bulk evolution over the course of the surveys. This follows from the expectation that at short time scales diffusivity dominates eddy evolution and estimates of an effective eddy diffusivity time scale fall between 80 and 290 days, varying with the strength of radial temperature and salinity gradients (Joyce 1977; Hebert et al. 1990; Ruddick and Richards 2003; Pelland et al. 2013). The time windows do not include all data collected during the surveys, but reflect all periods for which a reliable estimate of eddy structure and dynamics could be obtained (Fig. 3e). Variability in Cuddy translation speed corresponds to the variable length of each 5-day segment (Fig. 1). Time window five is highlighted and used as an example in the subsequent detailing of Cuddy structure and dynamics (Fig. 1, inset).

Since the analyses in this manuscript focus on time windows during which the eddy was sampled particularly densely, the results are not overly sensitive to the method used to determine the eddy position (appendix A). Use of an alternate method to estimate the eddy center during each window, which minimized the square angular differences between observed currents and those from an idealized azimuthally symmetric vortex, resulted in typical differences from the EKF smoother eddy position of $<1 \mathrm{~km}$. While this alternate method did not assume eddy azimuthal velocity structure, vortex strength, or vortex size, the results were still consistent with EKF estimates, but more sensitive to time window selection.

A cylindrical coordinate system was adopted in radius $r$ and height $z$ where relevant eddy quantities were assumed to be spatially independent of azimuthal angle (Elliott and Sanford 1986; D'Asaro 1988b; Pelland et al. 2013). Though the survey data in this study are not sufficiently dense to test the validity of this assumption, there was little evidence to contradict it in the individual transects that were examined (e.g., Fig. 2). Relevant eddy quantities, including spice, were then referenced to the moving center and associated with a radial distance (Fig. 4a). The 51 total profiles collected during time window five were almost entirely collected with their middepth point at $r<23 \mathrm{~km}$ (Fig. 4b); gliders were generally instructed to turn around and begin a new transect beyond this radius.

Referenced to the moving center, DAC vectors minus the EKF smoother estimated background mean flow $\mathbf{u}_{\text {back }}$ during each window (DAC $-\mathbf{u}_{\text {back }}$ ) and the azimuthal component of each of these vectors $\mathrm{DAC}_{\phi}$ were considered in analyzing eddy azimuthal symmetry and velocity structure (Fig. 4c). The differences between DAC $-\mathbf{u}_{\text {back }}$ and $\mathrm{DAC}_{\phi}$ may reflect uncertainty in the Cuddy center estimate, the mean background flow vector, aliased variability due to internal waves and tides, errors in the assumption of axisymmetry and spatially uniform background flow, and/or eddy radial currents. Vertical distance separating two isopycnals was also considered in analyzing Cuddy radial structure (Fig. 4c, inset). The chosen isopycnals $\left(\sigma_{\theta}=\right.$ $26.45 \mathrm{~kg} \mathrm{~m}^{-3}$ and $\sigma_{\theta}=26.65 \mathrm{~kg} \mathrm{~m}^{-3}$ ) vertically bound the Cuddy core, and their separation decreases to background levels with radius from the eddy core. Within each time window, $\mathrm{DAC}_{\phi}$ and a smoothed version, computed using an interpolation technique described below, increase with radius to a maximum speed at radii of $10-15 \mathrm{~km}$ before decaying back toward zero (Fig. 4d).

\section{c. Interpolation}

In each time window, one dimensional Gauss-Markov interpolation (Bretherton et al. 1976; Le Traon 1990) was used to map observed scalar quantities to a regular radial grid extending from the Cuddy center to $28 \mathrm{~km}$, in $0.5-\mathrm{km}$ intervals, on each vertically bin-averaged depth surface. This procedure was used to create radialvertical grids of $\theta, S, \sigma_{\theta}, \pi_{\theta}$, and $\mathrm{O}_{2}$, along with a radial grid of $\mathrm{DAC}_{\phi}$, for each of the 11 windows. Profiles completed within radii less than $2.5 \mathrm{~km}$ were copied, paired with negative radius values, and used to extend the observation domain from 0 to $-2.5 \mathrm{~km}$; this enforces the condition that isopycnal surfaces are horizontal and that eddy azimuthal currents are zero at the origin $(r=0)$.

The interpolation was performed using a horizontal decorrelation length scale of $15 \mathrm{~km}$. The choice of decorrelation length scale is intended to filter noise due to internal waves and high-frequency or wavenumber phenomena (Rudnick and Cole 2011) while retaining robust aspects of the Cuddy structure. By collapsing all glider profiles completed within a 5-day time window at various locations surrounding the Cuddy into one horizontal dimension, data density was greatly increased; this azimuthal averaging decreases the effect of noise, allowing a shorter smoothing scale than used previously to analyze eddies in this region (Pelland et al. 2013).

Use of this interpolation routine required a background field of all scalar quantities. For each window, profiles collected within 10 days of the midpoint time at a radius greater than $20 \mathrm{~km}$ and less than $35 \mathrm{~km}$ were 

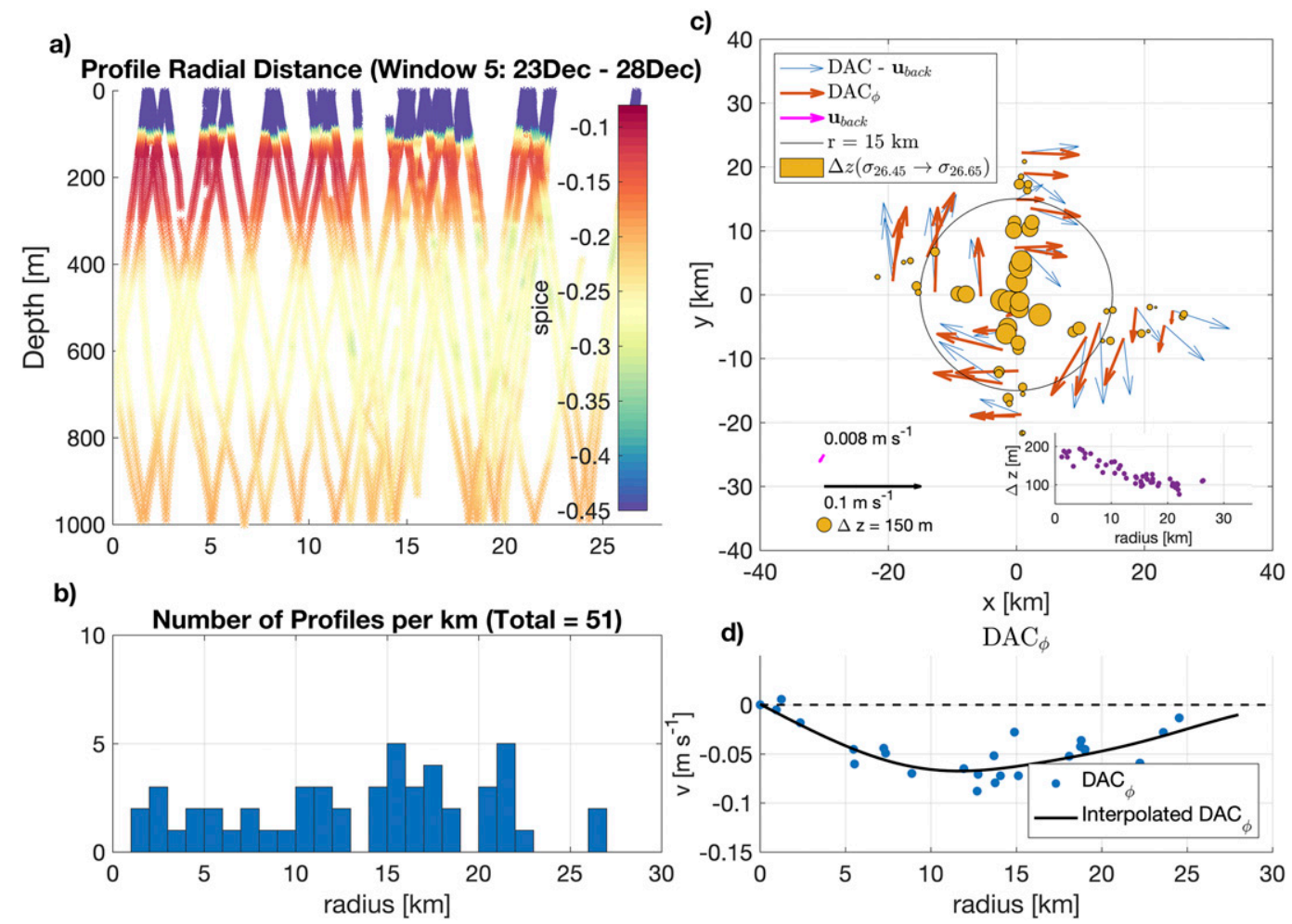

FIG. 4. Illustration of processing steps in example time window five. (a) Individual profiles of spice vs radius and depth made within this window and referenced to the time-varying eddy center. (b) Cast frequency vs radius. Gliders were instructed to turn around to begin a new transect near $\sim 30 \mathrm{~km}$. (c) Dive-climb cycle midpoint location and DACs in plan view, referenced to the Cuddy center. Blue arrows are glider-inferred DACs with an average background mean flow $\mathbf{u}_{\text {back }}$, estimated over the time window by the EKF, removed. Red arrows are their azimuthal component $\mathrm{DAC}_{\phi}$. The pink arrow is the average background flow estimated by the EKF over the time window. Yellow circles represent vertical separation of core-bounding isopycnals $\left(\sigma_{\theta}=26.45 \mathrm{~kg} \mathrm{~m}^{-3}\right.$ and $\left.\sigma_{\theta}=26.65 \mathrm{~kg} \mathrm{~m}^{-3}\right)$ estimated on each vertical profile. The size of the circle linearly scales with the magnitude of separation. The inset at the bottom right is the true separation as a function of radius. (d) $\mathrm{DAC}_{\phi}$ (blue dots: individual cycles, black: interpolated values) vs radius.

averaged along depth surfaces to yield background profiles of $\theta, S, \sigma_{\theta}$, and $\mathrm{O}_{2}$. These conditions were chosen in order to yield profiles with no observed increase from background levels, like those observed by SG108, in separation between isopycnals bounding the Cuddy core and spice anomaly at the Cuddy core depth. Differing background profiles were estimated for each window because, as the Cuddy translated, it moved through cross-shore horizontal gradients of temperature and salinity evident in SG108 transects (Fig. 3).

The results of the interpolation procedure are gridded, spatially smoothed scalar and velocity fields versus $r$ and $z$ (e.g., $\pi_{\theta}$ in Fig. 5a). Within these gridded fields, the core region is defined as extending in radius from $0 \mathrm{~km}$ to $r_{v_{\max }}$, the radius of maximum velocity, which will be defined in the next section. The far field is assumed to feel little influence of the Cuddy while a transition region includes the area of interaction between the interior Cuddy core and exterior background waters (Fig. 5a).

\section{Analysis}

\section{a. Estimates from interpolated fields}

The gradient-wind horizontal momentum balance appropriate for describing intense submesoscale and mesoscale eddies includes a nonnegligible centripetal term (Holton 1972; McWilliams 1985; D'Asaro 1988b; Hebert et al. 1990; Schultz Tokos and Rossby 1991; Martin et al. 2009; Pelland et al. 2013; Bosse et al. 2016). In cylindrical coordinates this is defined as

$$
\frac{\partial \Phi}{\partial r}=f v+\frac{v^{2}}{r}
$$

where $\Phi$ is geopotential, $v$ is the azimuthal velocity, and $f$ is the local Coriolis parameter. For each analysis 

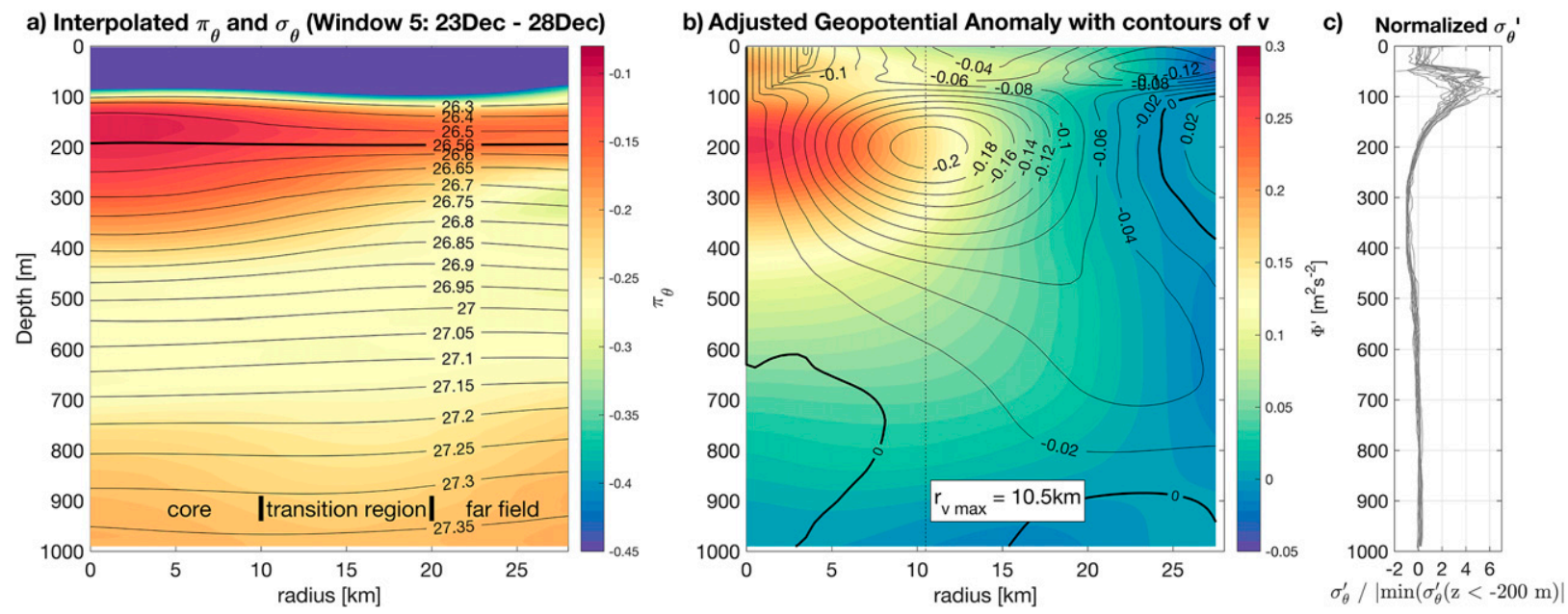

FIG. 5. (a) Interpolated spice $\pi_{\theta}$ (colors) and potential density $\sigma_{\theta}$ (black contours) for time window five. (b) Geopotential anomaly $\Delta \Phi$, relative to a far field absolute geopotential background profile, with absolute gradient wind azimuthal velocity $v_{\phi}$ contoured in black (section 4a). Negative values of velocity reflect anticyclonic, clockwise circulation in cylindrical polar coordinates. (c) Sixteen density anomaly profiles from time window five and at radii $<11 \mathrm{~km}$. Anomalies are computed relative to an average density profile computed using profiles with radii $>15 \mathrm{~km}$. Profiles are normalized by the absolute value of the density anomaly minimum at $z<-200 \mathrm{~m}$.

window, we calculated absolute gradient-wind velocity fields $v(r, z)$ referenced to the estimated azimuthal DAC as follows: using the interpolated density field as described above, geopotential was first calculated relative to a deep level of no motion $p_{0}=980 \mathrm{dbar},(1)$ was solved using this relative geopotential, and then, at each radial grid station, the radial gradient of the geopotential at the deep reference level was iteratively adjusted until the vertical average of $v$ from (1) at each station matched the interpolated $\mathrm{DAC}_{\phi}$ at that station (Pelland et al. 2013). The radial geopotential gradient at the reference level was then integrated radially from the origin to $r=28 \mathrm{~km}$ to produce a profile of absolute geopotential at the reference level, defined to within an arbitrary constant. This radial profile was added to the relative geopotential field at each depth level to give the absolute geopotential $\Phi$. This is similar to the method of Bosse et al. (2016, their appendix A) for determining the absolute depth-averaged radial pressure gradient by referencing gradient-wind velocities to DAC. Using the absolute gradient-wind velocity field, the vertical component of relative vorticity $\zeta$ was calculated according to

$$
\zeta=\frac{\partial v}{\partial r}+\frac{v}{r}
$$

The geopotential anomaly $\Delta \Phi$, a measure of Cuddy strength, was found by subtracting from the absolute geopotential a far-field profile defined as the average along depth surfaces within the range $22 \leq r \leq 28 \mathrm{~km}$.
This far-field profile was removed from the entire field with the result defined as the geopotential anomaly $\Delta \Phi$ associated with the Cuddy (Fig. 5b). In analysis window five, the maximum geopotential anomaly is evident at the Cuddy core depth in the main pycnocline.

The Cuddy had clockwise, anticyclonic circulation; in the example fifth window, the maximum azimuthal currents were $0.21 \mathrm{~m} \mathrm{~s}^{-1}$ at $200-\mathrm{m}$ depth and a radial distance $r_{v_{\max }}=10.5 \mathrm{~km}$ (Fig. 5b). Here $r_{v_{\max }}$ is defined as the radial distance, in each analysis window, along the depth surface of maximum geopotential anomaly at which the strongest azimuthal currents are found. This radius is used to delineate the Cuddy core from the exterior. A second horizontal length scale $r_{v=c}$ was also calculated as the outermost radial station at the core depth at which the azimuthal velocity $v$ is equal to or greater than the eddy translation speed $c$. At radii close to but less than $r_{v=c}$, azimuthal currents exceed the translation speed. This identifies a boundary within which water is trapped and can be transported laterally within the eddy core (Early et al. 2011).

\section{b. Model-derived dynamics}

Note that the geopotential, velocity, and vorticity estimates derived from the interpolated fields assume no Cuddy structure beyond azimuthal symmetry. For some quantities, it is convenient to further assume a radial-vertical eddy structure (an eddy "model") and fit this to the data in each analysis window. We assume that Cuddy density and geopotential structure can be compactly described by a separable structure, taken 
as a product of radial and vertical functional forms. The vertical structure of nondimensionalized eddy density anomaly profiles (Fig. 5c) do not demonstrate evidence of radial dependence and support the assumption of separability. The model geopotential anomaly $\widehat{\Delta \Phi}$ takes the form:

$$
\widehat{\Delta \Phi}=\left\{\begin{array}{lll}
A\left[\frac{9}{8}-\left(\frac{r}{\lambda}\right)^{2}\right] \exp \left[-\frac{1}{8}-\left(\frac{z-z_{0}}{h}\right)^{2}\right], & \text { for } & r<\frac{\lambda}{2 \sqrt{2}} \\
A \exp \left[-\left(\frac{r}{\lambda}\right)^{2}-\left(\frac{z-z_{0}}{h}\right)^{2}\right], & \text { for } & r \geq \frac{\lambda}{2 \sqrt{2}}
\end{array}\right.
$$

where $A, \lambda$, and $h$ are the Cuddy strength, radial scale, and vertical scale, respectively. For each of the 11 analysis windows the Cuddy core depth $z_{0}$ and eddy center are defined as the depth at $r=0$ where $\Delta \Phi$ is a maximum. The model scale and strength parameters were then estimated by minimizing the squared error between model and observed $\Delta \Phi$. The piecewise model form (3) was chosen to include an inner region $r \leq \lambda /(2 \sqrt{2})$ for which azimuthal speed varies nearly linearly with radius to approximate solid body rotation. At greater radii, the Gaussian form was chosen to describe smoothly varying flow that peaks at $r \approx 0.6 \lambda$ (McWilliams 1985; Pelland et al. 2013). For simplicity, the vertical structure is assumed to be symmetric about the core depth.

In addition to velocity and vertical vorticity fields computed directly from the interpolated grids as described above, these quantities were also computed from (3) for comparison. The resulting model fields, $\hat{v}$ and $\hat{\zeta}$, are independent of background flow and describe idealized monopole SCV structure dependent only on the three parameters $A, \lambda$, and $h$. An additional benefit of (3) is that quantities unable to be fully specified from the interpolated fields, such as potential vorticity at the eddy center, can be estimated from the model. Potential vorticity (PV) is computed as (D'Asaro 1988b; Martin et al. 2009)

$$
\mathrm{PV}(r, z)=(\hat{\zeta}+f) \frac{N^{2}}{g}+\frac{1}{\rho_{0}} \frac{\partial \hat{v}}{\partial z} \frac{\partial \sigma_{\theta}}{\partial r}
$$

The first term in (4), describing the PV vertical component, is the product of the vertical component of absolute vorticity with the buoyancy frequency $N^{2} \equiv-\left(g / \rho_{0}\right) \partial \sigma_{\theta} / \partial z$, divided by the gravitational acceleration $g$, where $\rho_{0}=1025 \mathrm{~kg} \mathrm{~m}^{-3}$ is a reference density. The second term describes twisting and tilting. This estimate of PV combines the modeled geopotential anomaly, through velocity and vorticity, with the interpolated density field, which is used to estimate $N^{2}$.

Velocity and vorticity Rossby numbers for the Cuddy were computed as $\mathrm{Ro}_{v}=2 v_{\max } /\left(f r_{v_{\max }}\right)$ and $\mathrm{Ro}_{\zeta}=\left|\zeta_{\left(0, z_{0}\right)}\right| / f$, respectively (D'Asaro 1988b). The velocity Rossby number is the ratio of relative to planetary vorticity and is a measure of the importance of the nonlinear advection term in (1). The factor two arises from writing the momentum balance in terms of the horizontal density gradient and vertical azimuthal velocity shear. Note, $v_{\text {max }}$ is defined as the greatest azimuthal speed at the core depth and is thus positive. The vorticity Rossby number, directly relates the Cuddy core to planetary vorticity. Values greater than one reflect a core susceptible to inertial instability (D'Asaro 1988b). The Burger number was additionally computed to explore aspect ratio adjustment. It is defined as the square of the ratio of the baroclinic radius of deformation to the horizontal length scale of the flow $\left\{\mathrm{Bu}=\left[N_{0} h /(f \lambda)\right]^{2}\right.$, where $N_{0}$ is the background stratification averaged between $160 \mathrm{~m}$ and $240 \mathrm{~m}$ \}. Its value through time describes how an eddy adjusts as it translates into regions of different background stratification and/or planetary vorticity. Initial horizontal and vertical length scales are set by formation processes and the background state, but as an eddy propagates it is expected to adjust to a stable state and shape within a range $0.05<\mathrm{Bu}<1$ (McWilliams and Gent 1986).

Total available potential energy (APE) and kinetic energy (KE) in each analysis window were computed as (D'Asaro 1988b; Hebert et al. 1990)

$$
\begin{aligned}
\mathrm{APE} & =\int_{z}^{0} \int_{0}^{2 \pi} \int_{0}^{28 \mathrm{~km}} \frac{1}{2} \hat{\rho} N_{b}^{2} \xi^{2} r d r d \theta d z, \\
\mathrm{KE} & =\int_{z}^{0} \int_{0}^{2 \pi} \int_{0}^{28 \mathrm{~km}} \frac{1}{2} \rho_{0} \hat{v}^{2} r d r d \theta d z,
\end{aligned}
$$

where $\hat{\rho}$ is the observed background density profile plus the model density anomaly found using (3). Here, the background buoyancy frequency profile $N_{b}^{2}$ is calculated using the background density profile, and $\xi$ is the vertical displacement of each isopycnal from its background level. To the extent that any eddy velocity and density anomalies are nonzero deeper than the maximum depth of glider profiling, these expressions will underestimate the true APE and KE. Full-depth sections of Cuddies are rare, but previous observations 
have shown isopycnal displacements and baroclinic velocities limited to the upper $1200 \mathrm{~m}$ (Simpson et al. 1984) or shallower (Huyer et al. 1984). The near-zero nondimensionalized density anomalies (Fig. 5c) and low azimuthal velocities observed at $1000 \mathrm{~m}$ (Fig. 5b) in this study are consistent with these observations.

\section{c. Uncertainty estimates}

Uncertainties in the estimates described above due to errors in the eddy center location were estimated using Monte Carlo sampling. In each analysis window, 100 iterations were performed, in each of which the Cuddy center position was adjusted. For each iteration, randomly drawn, normally distributed $x$ and $y$ perturbations, with covariance structure specified by the error covariance matrix output by the EKF smoother at the middle time point of each window (appendix A), were used to shift the position of all center points within that window. The interpolation technique was then reapplied for each iteration and all dynamic quantities were recomputed; $95 \%$ confidence intervals were computed as two standard deviations of each quantity across the 100 iterations. This procedure conservatively assumed that center estimate errors between time points were dependent within each time window.

\section{d. Temporal change}

Temporal changes in Cuddy dynamic fields and bulk tracer properties were analyzed by comparing average properties early during the tracking period and after the Cuddy's rapid translation northward. Quantities were averaged within "early" and "late" analysis windows; late windows were defined as those following the onset of rapid poleward propagation (9 January). This distinction results in seven early and four late analysis windows (corresponding roughly to the first and second halves of the observation period). The statistical significance of differences in means between these two periods was assessed using a Student's $t$ test (von Storch and Zwiers 2003); changes between early and late were considered significant if the null hypothesis of no difference in means between the two time periods was rejected at $95 \%$ confidence.

\section{Results}

\section{a. Characteristic scales}

As the Cuddy translated northward, the background stratification at its core depth decreased by $9 \%$ and the Cuddy core isopycnal $\sigma_{\theta}=26.56 \mathrm{~kg} \mathrm{~m}^{-3}$ deepened, along with the depth of maximum geopotential anomaly and the depth of maximum azimuthal velocity (Fig. 6a). The core isopycnal was selected as the isopycnal that, over all snapshots, was least vertically deflected from the background at radii less than $10 \mathrm{~km}$. Its depth was estimated for each snapshot using the five glider profiles closest to the Cuddy center. These depths were compared against far-field estimates made using the five profiles completed farthest from the Cuddy center. Throughout the tracking period, the core isopycnal deepened everywhere; core and far-field depth estimates were positively correlated with a square correlation coefficient of over 0.9 .

As this deepening occurred, the background buoyancy frequency at the Cuddy core depth and the Cuddy core buoyancy frequency converged (Fig. 6a). While the horizontal length scale $\lambda$ and radius of maximum velocity $r_{v_{\max }}$ did not significantly change between early and late periods, the radius at which azimuthal currents matched eddy translation speed $r_{v=c}$ decreased by $20 \%$ beginning 6 January (Fig. 6b). Its decrease in early January is consistent with a concurrent increase in background flow measured by SG108 and estimated by the EKF. The decrease in $r_{v=c}$ during the second half of the survey period is most evident in two estimates where translation speed is strongest (12 and 27 January).

The magnitude of the geopotential anomaly $\widehat{\Delta \Phi}$ decreased by $20 \%$ from the first to the last half of the record (Fig. 7a), while $\lambda$ did not significantly change (Fig. 7b) and $h$ increased by $38 \%$ (Fig. 7c). These changes occurred as background stratification weakened by $9 \%$ between early and late averages. Coincident with these changes, the eddy core stratification increased from $30 \%$ to $55 \%$ of local background values (Fig. 6a).

\section{b. Kinematics and dynamics}

Modest temporal changes in eddy rotation and stratification over the observing period are evident from azimuthal velocity, vorticity, and potential vorticity radial profiles along the core depth (Fig. 8). Mapped velocity profiles (Fig. 8a) reveal a nearly linear gradient, indicative of solid body rotation, only for $r \leqq r_{v_{\max }} / 2$ where $r_{v_{\max }}=7-10 \mathrm{~km}$. At larger radii, the profiles follow a roughly Gaussian shape providing the motivation for the model form in (3).

Both model and directly estimated peak azimuthal velocity decreased modestly over time from a maximum magnitude of $0.23 \mathrm{~m} \mathrm{~s}^{-1}$ in the interpolated field in window two to $0.19 \mathrm{~m} \mathrm{~s}^{-1}$ in window eleven (early average peak speed $=0.21 \mathrm{~m} \mathrm{~s}^{-1}$, late average peak speed $=0.175 \mathrm{~m} \mathrm{~s}^{-1}$ ). The magnitude of the ratio of the core relative vorticity to $f$ decreased more dramatically from an early average of 0.69 to a late average of 0.46 . The radial profile shape of relative vorticity normalized by $f$ reflects the choice of a model permitting an inner core in solid body rotation. Validation of this model choice is further confirmed by the time-averaged vorticity profile 
a)

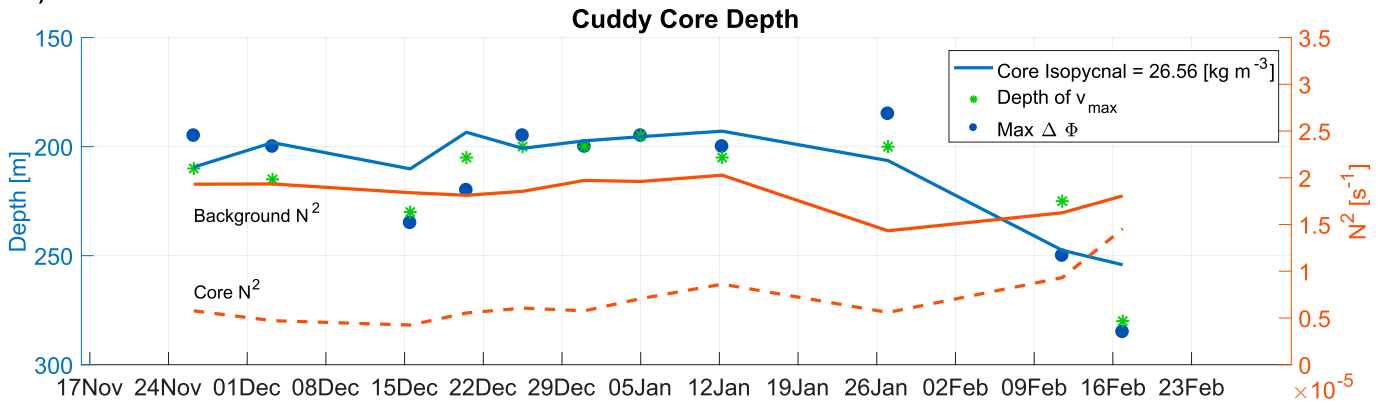

b)

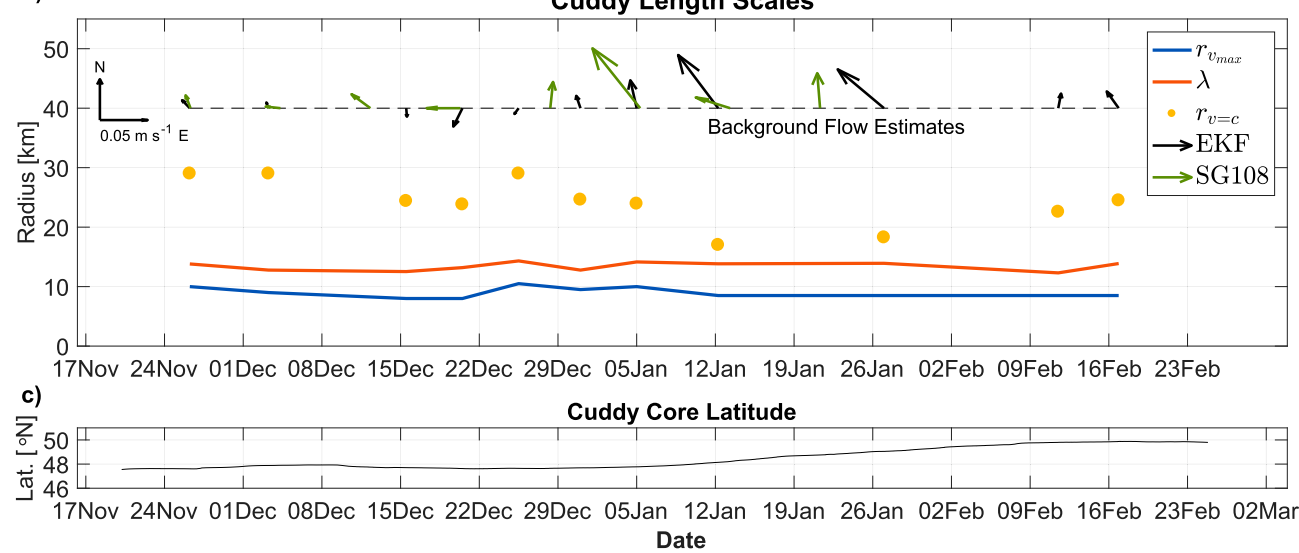

FIG. 6. (a) Depth of the target Cuddy core isopycnal (blue line), estimated maximum geopotential anomaly (blue circles), and maximum azimuthal velocity (green stars) vs time. Referenced to the second (right) vertical axis are background (solid red) and Cuddy core (dashed red) buoyancy frequency at the core depth vs time. (b) Cuddy horizontal scale indices: radius of maximum azimuthal current $r_{v_{\max }}$ (blue curve), model radial scale $\lambda$ (red curve), and radius at which azimuthal current matches translation speed (yellow circles) vs time. Average eddy translation vectors estimated from the EKF within each analysis window are in black. Green vectors are independent estimates of the background flow from SG108, averaged over the same time windows and cross-shore domain occupied by the Cuddy. (c) Cuddy latitude vs time.

from the interpolated fields where at small radii the slope of vorticity flattens (Fig. 8b). In time, vorticity weakened particularly within the core, consistent with the weakening of the velocity field. $\mathrm{PV}$ at a radius of $0 \mathrm{~km}$ and at the core depth increased from $11 \%$ of the background value to $20 \%$ by the end of the survey. This is seen as an increase (decrease in magnitude) in PV anomaly, a ratio defined as $(\mathrm{PV}-\mathrm{PV}$ ff $) / \mathrm{PV}_{\mathrm{ff}}$, where $\mathrm{PV}_{\mathrm{ff}}$ refers to the farfield $\mathrm{PV}$ defined as the average $\mathrm{PV}$ at radii greater than $20 \mathrm{~km}$ (Fig. 8c).

Velocity and vorticity Rossby numbers significantly decreased between the early and late period (Figs. 9a,b), reflecting an increase in $f$ (by $3 \%$ ) and a decrease in peak azimuthal velocity. The Burger number did not change significantly between early and late periods (Fig. 9c). While the aspect ratio $(h / \lambda)$ increased by the end of the survey period (Fig. 7), this was somewhat compensated by a decrease in the ratio of the background buoyancy frequency to Coriolis frequency. Cuddy total mechanical energy was roughly equally partitioned between APE and KE and while APE significantly decreased by $45 \%$ between early and late averages, KE did not significantly decrease (Fig. 10).

\section{c. Bulk tracer evolution}

Temperature and salinity anomalies along isopycnals within the cuddy core, and their contrast with cool, fresh offshore water in the Washington coastal region, are evident when viewed in $\theta / S$ space (Fig. 11). Background profiles, averaged between November and January and collected by SG108, indicate spice decreases with distance offshore within the thermocline (profiles collected offshore are cooler and fresher). A pair of profiles collected within the Cuddy when it was located more than $100 \mathrm{~km}$ offshore indicate its core is even more spicy, as evidenced by warm and salty waters found between $\sigma_{\theta}=26.4 \mathrm{~kg} \mathrm{~m}^{-3}$ and $\sigma_{\theta}=27 \mathrm{~kg} \mathrm{~m}^{-3}$. The relatively high values of spice within the Cuddy suggest that it formed at a location equatorward of the SG108 transect and distinguish it from surrounding poleward waters. 


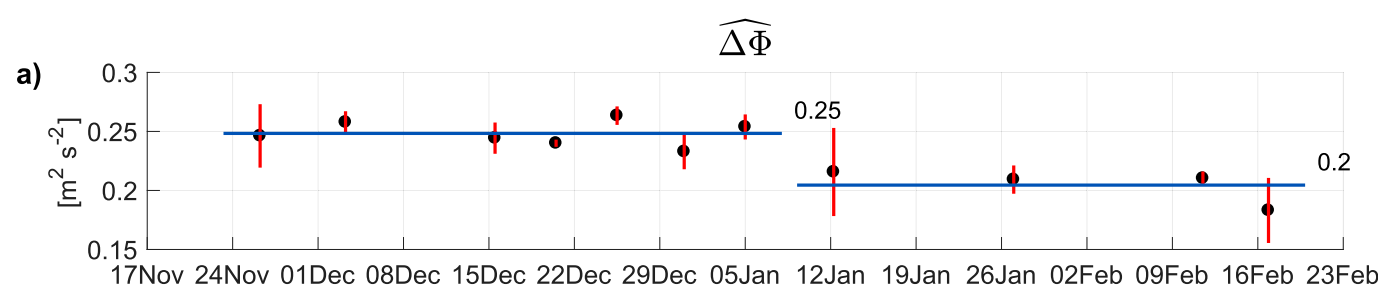

b)

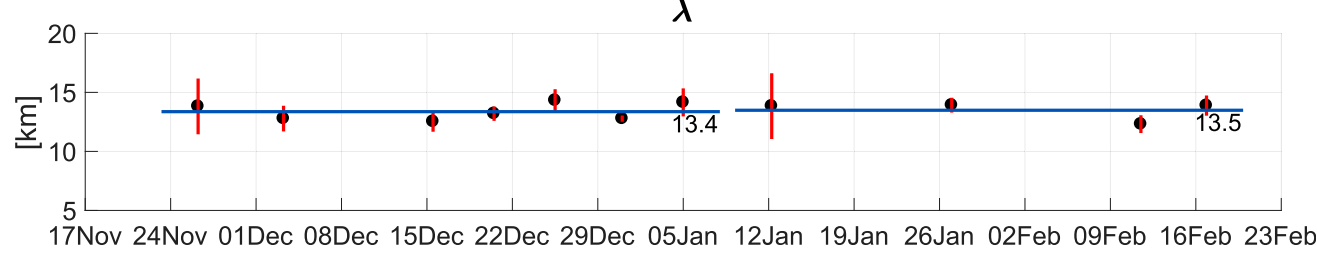

c)

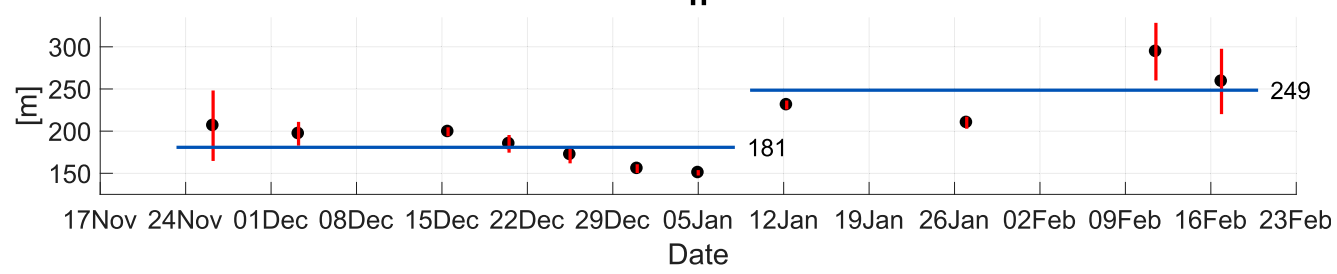

FIG. 7. Model estimated parameters scattered in black as a function of time: (a) $\widehat{\Delta \Phi}$, (b) $\lambda$, and (c) $h$; as defined in section 4b. Vertical red bars are $95 \%$ confidence intervals, and horizontal blue lines are early- and late-period averages.

Evolution of spice, dissolved oxygen, and potential vorticity within the Cuddy can be seen from a sequence of $r-z$ sections from representative snapshots (Fig. 12). As indicated by the time series of model scale parameters (Fig. 7), azimuthal velocity weakens and core depth deepens through the course of observations accompanied by a decrease in spice (Fig. 12, left panels). Over the same period, dissolved oxygen change within the core is indiscernible (center panels), but potential vorticity weakens modestly, as measured by the core volume contained within the $\mathrm{PV}=1 \times 10^{-10} \mathrm{~m}^{-1} \mathrm{~s}^{-1}$ contour (right panels), the same volume used to estimate average spice and dissolved oxygen within the core (Fig. 12, bottom panels). When early and late averages of quantities in this core region are compared, core spice decreases from -0.13 to -0.15 (a statistically significant change), $\mathrm{O}_{2}$ by $1 \%$ (not significant), and core volume by $6 \%$ (not significant).

Despite average $\mathrm{O}_{2}$ remaining essentially constant, by the survey's end, core oxygen variance had increased by over an order of magnitude (Fig. 13a). Vertical profiles within each time window were ordered by radial distance and oxygen variance computed between $26.52 \mathrm{~kg} \mathrm{~m}^{-3}<\sigma_{\theta}<26.6 \mathrm{~kg} \mathrm{~m}^{-3}$ by averaging over $6 \mathrm{~km}$ overlapping radial bins. The use of a strict vertical bound intends to highlight horizontal, not vertical, variations. Initially, core oxygen variance was very low, $<10\left(\mu \mathrm{mol} \mathrm{kg}^{-1}\right)^{2}$ at $r<10 \mathrm{~km}$. Outside the core at larger radii, variance was an order of magnitude greater, peaking at $r=17 \mathrm{~km}$, and again decreasing to modest exterior values beyond $r>18 \mathrm{~km}$ (Fig. 13a). This initial radial profile is consistent with an inner homogenous core, a transition region of mixing waters, and a more uniform background outside the Cuddy. Over time the radius of maximum variance migrated toward smaller radii and by the end of the tracking period, core oxygen variance had increased tenfold. The radial distribution of spice variance (Fig. 13b) displayed a qualitatively similar evolution in time; by the end of the survey period, core spice variance increased by nearly a factor of three.

Vertical profiles of spice at each radial grid station and for each snapshot were interpolated to a density grid to consider changes in core spice. A far-field average profile for each snapshot was removed and resulting spice anomaly profiles at the Cuddy center, $r=0 \mathrm{~km}$ are considered (Fig. 14). From late November through midJanuary a relatively compact positive roughly triangular anomaly characterized Cuddy spice. Added for reference is the core isopycnal, as well as shallower $\sigma_{\theta}=26.3 \mathrm{~kg} \mathrm{~m}^{-3}$ and deeper $\sigma_{\theta}=27 \mathrm{~kg} \mathrm{~m}^{-3}$ isopycnals bounding the core (Fig. 14). Starting in late January and continuing through the last three snapshots, spice anomaly in the core decrease to more uniform values across the range of core densities.

Using salt, a more easily interpretable tracer, we computed the available salt anomaly (ASA; Pelland et al. 2013). Instead of using a background profile for 

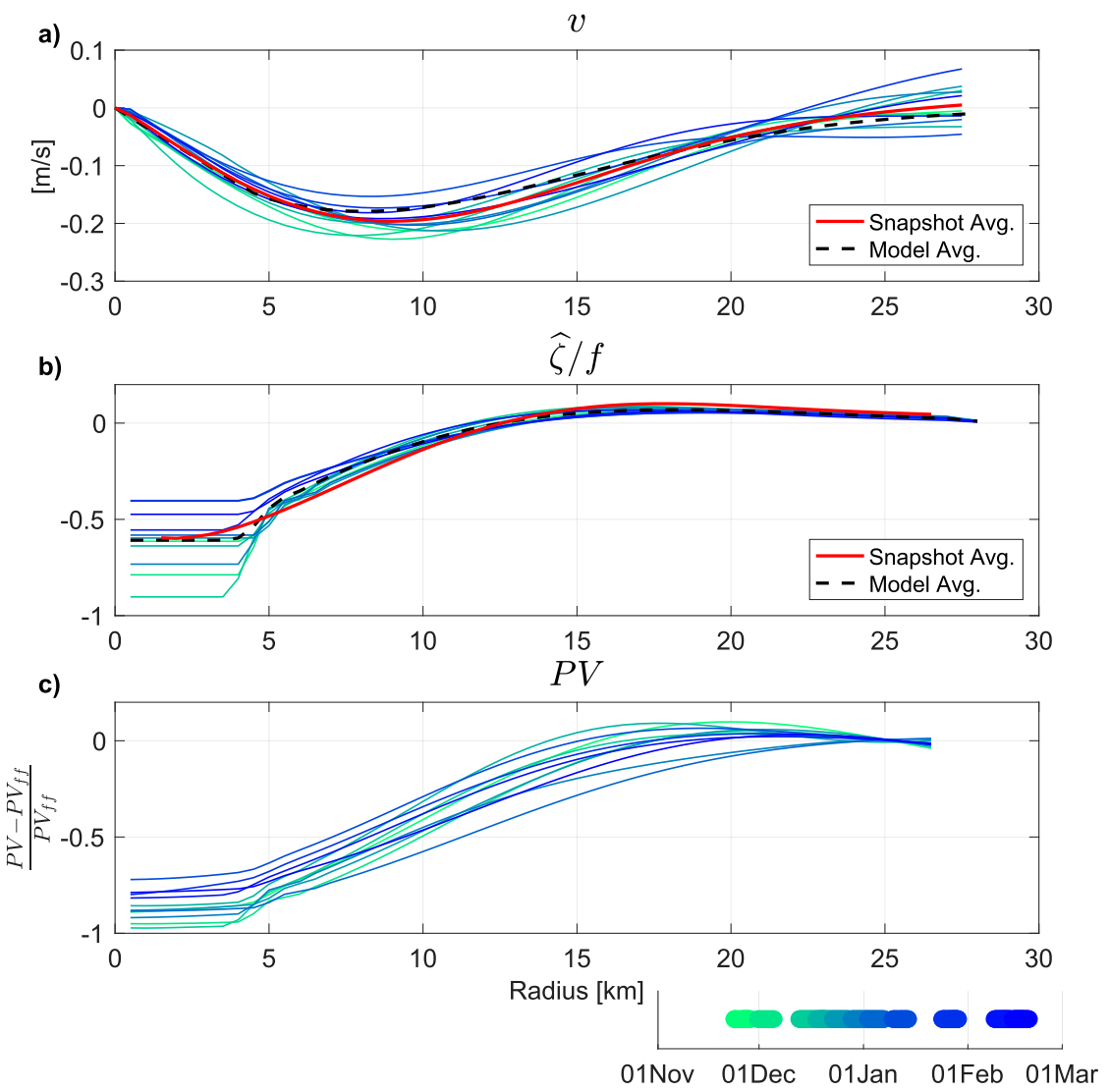

FIG. 8. (a) Radial profiles of azimuthal velocity derived from the interpolated fields $v_{\phi}$ at the Cuddy core depth $\left(z=z_{0}\right)$ in each analysis window. Color corresponds to the window time (advancing from green to blue; inset). Solid red profile is an average of these. The dashed black line is an average of model-estimated velocity profiles $\hat{v}$. (b) Radial profiles of relative vorticity, in this case estimated from the model $\hat{\zeta}$, divided by the local Coriolis parameter $f$ at the Cuddy core depth; individual and average profiles are colored as in (a). (c) Radial profiles of PV anomaly at the core depth, subtracted from and normalized by a far-field average.

each window, one reference profile, collected at the survey midpoint, was used such that anomalies could be compared against each other through time. ASA is defined here as

$$
\mathrm{ASA}=\int_{z_{1}}^{z_{2}} \int_{0}^{2 \pi} \int_{0}^{\lambda} 0.001 \rho_{0}\left(S_{\sigma_{\theta}}-S_{\sigma_{\theta}}^{\mathrm{ref}}\right) r d r d \theta d z
$$

where $S_{\sigma_{\theta}}$ and $S_{\sigma_{\theta}}^{\text {ref }}$ correspond to salinity and a reference profile all gridded on density surfaces. A volume integral was taken between vertical levels $z_{1}$ and $z_{2}$, set by the corresponding depths of $\sigma_{\theta}=26.3 \mathrm{~kg} \mathrm{~m}^{-3}$ and $\sigma_{\theta}=$ $27 \mathrm{~kg} \mathrm{~m}^{-3}$. ASA significantly decreased by $27 \%$ between early and late periods (Fig. 14, bottom panel).

\section{d. Vertical fine structure}

Occurrences of vertical fine structure, suggestive of lateral exchange among waters of different spice between eddy core and exterior regions, were observed with increasing frequency in time throughout the survey period. Within the Cuddy core, $\theta / S$ profiles are self-similar and differ little from one another (Fig. 15, left panel). Outside the core, $\theta / S$ curves are generally cooler and less salty, but often irregularly vary from these to spicier conditions, indicative of intrusive behavior along selected potential density surfaces (Fig. 15, right panel).

Shcherbina et al. (2009) characterized thermohaline lateral intrusions by spice curvature in $\theta / S$ space, an approach adopted here to address Cuddy spice variability. Two derivatives of spice with respect to density were calculated after smoothing each glider profile on a density grid using a triangular filter with a half-width of $0.03 \mathrm{~kg} \mathrm{~m}^{-3}$. Local maxima and minima in curvature identify the position, on a density grid, of intrusive features on the order of tens of meters. A threshold of two standard deviations, found using all curvature measurements within 

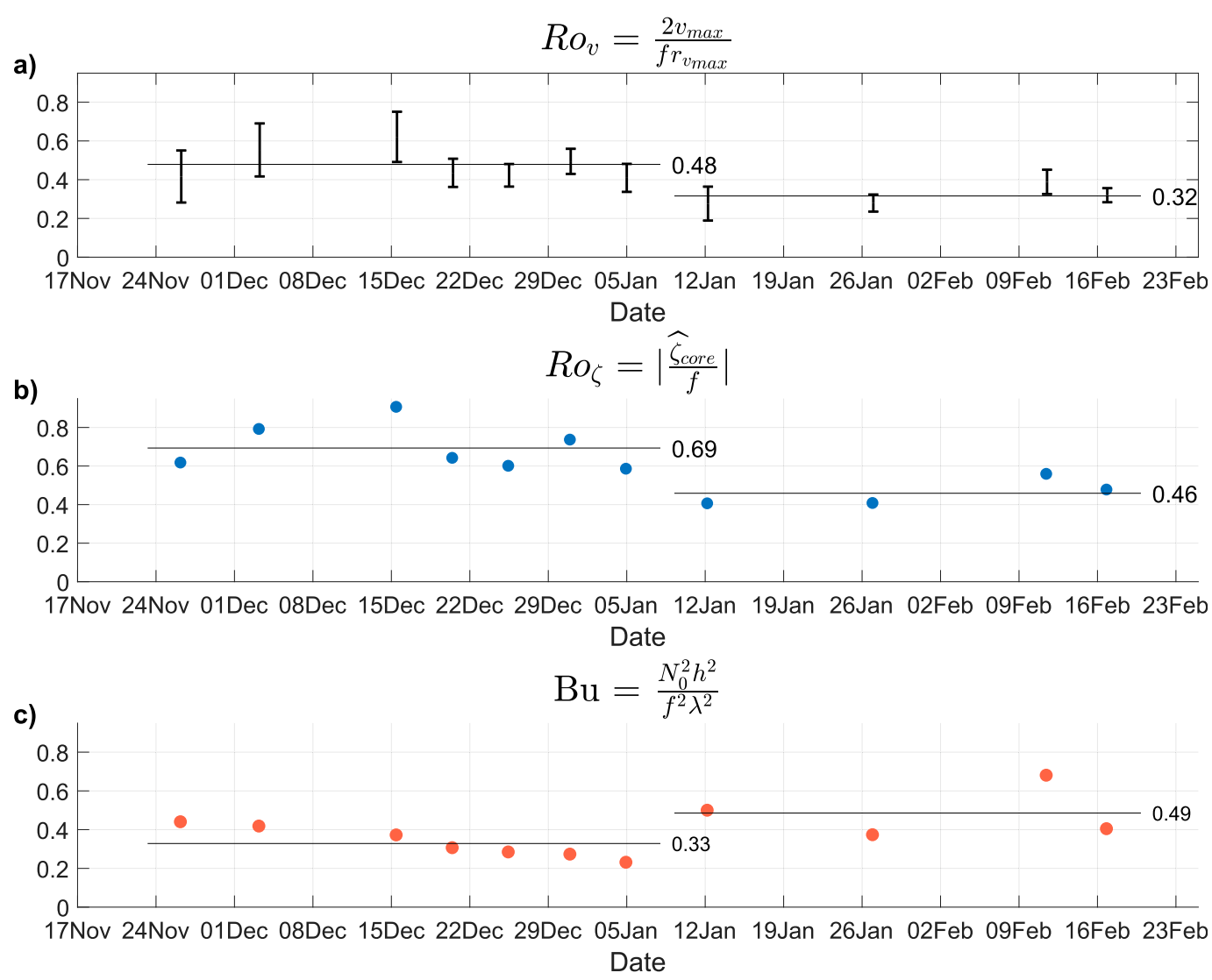

FIG. 9. (a) Rossby number $\mathrm{Ro}_{v}$, (b) vorticity Rossby number $\mathrm{Ro}_{\zeta}$, and (c) Burger number computed using modelderived velocity fields and dimensions vs time. Error bars indicate $95 \%$ confidence intervals, while horizontal lines indicate early (first 7 snapshot estimates) and late (last 4) averages.

each Cuddy snapshot, was then used to identify intrusions relative to the Cuddy core PV boundary and well above levels of background variability (Fig. 16).

Interleaving events are most common at the base of the mixed layer $(\sim 80 \mathrm{~m})$, somewhat less common at deeper depths, and even rarer near the core depth (Fig. 16). As the survey progressed, the occurrence of interleaving events decreased at larger and increased at smaller radii at depths both shallower and deeper than the Cuddy core. Inferred interleaving events ultimately became common at radii less than the eddy radial scale, suggesting that intrusions reached core waters. The percentage of intrusions found within each time window at radii less than $\lambda$ increased from $19 \%$ in the early period to $72 \%$ in the late period (Fig. 16, bottom right panel).

\section{Discussion of eddy translation and erosion}

The persistent survey of a Cuddy over the course of three months revealed modest structural changes. Consistency in eddy visual appearance and the core depth estimate, across all analysis windows, suggests the successful tracking of the same feature. The consistent observation of southeastward flow and isopycnal separation consistent with ambient, offshore conditions (Fig. 4c) on its inshore edge rule out the interpretation of this feature as a meander of the undercurrent rather than an isolated vortex.

The deepening of the core isopycnal, both within the eddy (Fig. 6a) and in the far field, is indicative of the eddy propagating into waters with a background density structure different from that closer to its formation site. Isolated subsurface eddies like that observed in this study are expected to propagate vertically across potential density and neutral surfaces due to compressibility effects, but these effects are likely very small [movement of $\mathscr{O}(1) \mathrm{m}$ given conditions in this study; McDougall 1987] relative to the observed vertical displacements.

A unique aspect of this study was the Cuddy's rapid translation to higher latitude, which likely decreased the isolation of core waters in addition to transporting the eddy into different background conditions. Within one week in early January, translation speeds more than doubled to over $0.08 \mathrm{~m} \mathrm{~s}^{-1}$. Compared to speeds 


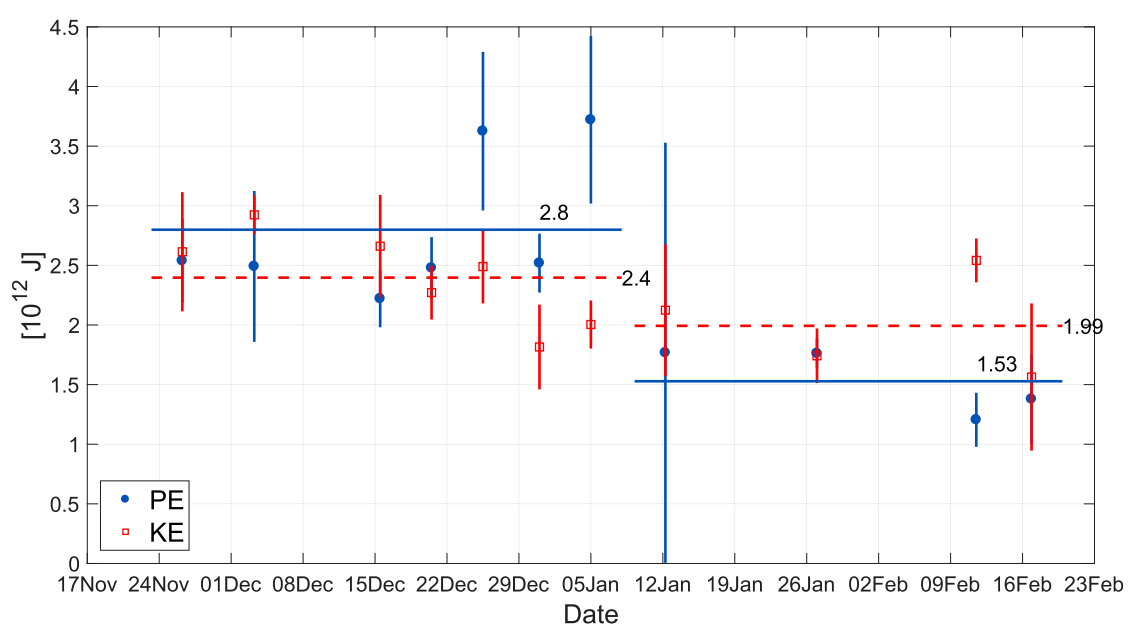

FIG. 10. Volume integrated available potential (PE; blue) and kinetic (KE; red) energy for each snapshot. Vertical bars are $95 \%$ confidence limits accounting for center error estimates. Horizontal lines are early and late time averages with average values labeled.

consistently less than $0.04 \mathrm{~m} \mathrm{~s}^{-1}$ over the preceding three weeks, this abrupt alongshore propagation contrasts with the expectation of steady offshore movement as described by Collins et al. (2013), with the exception of a single float tracked off of the Washington coast that similarly exhibited poleward movement. The retention of the eddy within the nearshore region is also similar to the modeled Cuddy trajectory of Torres and Gomez-Valdes (2017) in the southern CCS. In applying an asymptotic theory of SCV motion, Dewar and Meng (1995) state that the beta drift term appears at the next order expansion following terms describing an eddy and a mean flow. This suggests that eddy self-translation due to the beta effect alone can be overwhelmed by a mean flow.

Off of the Washington coast in early winter, as the Cuddy experienced surface and subsurface northward background flows, its trajectory was consistent with the expectation of flow following contours of potential vorticity $(\approx f /$ water depth). Between approximately 15 and 30 December, a period of southwestward

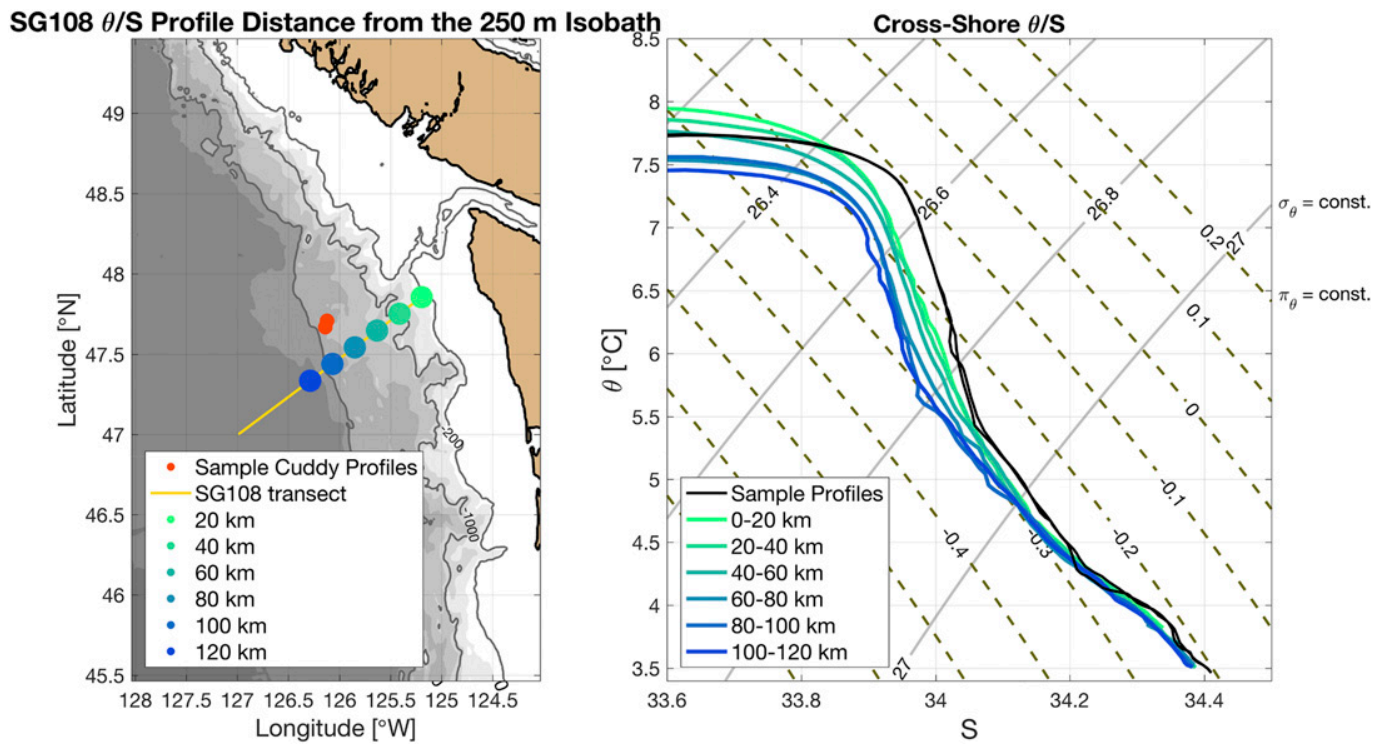

FIG. 11. Cross-shore average potential temperature/salinity $(\theta / S)$ profiles collected by SG108 between 20 Nov 2013 and 20 Jan 2014. (left) The location of the average profiles as well as the position of the Cuddy during mid December. As distance offshore increases, color goes from green to blue. (right) The $\theta / S$ curves are colored accordingly. Two profiles made through the Cuddy core are in red in the left panel and black in the right panel. 

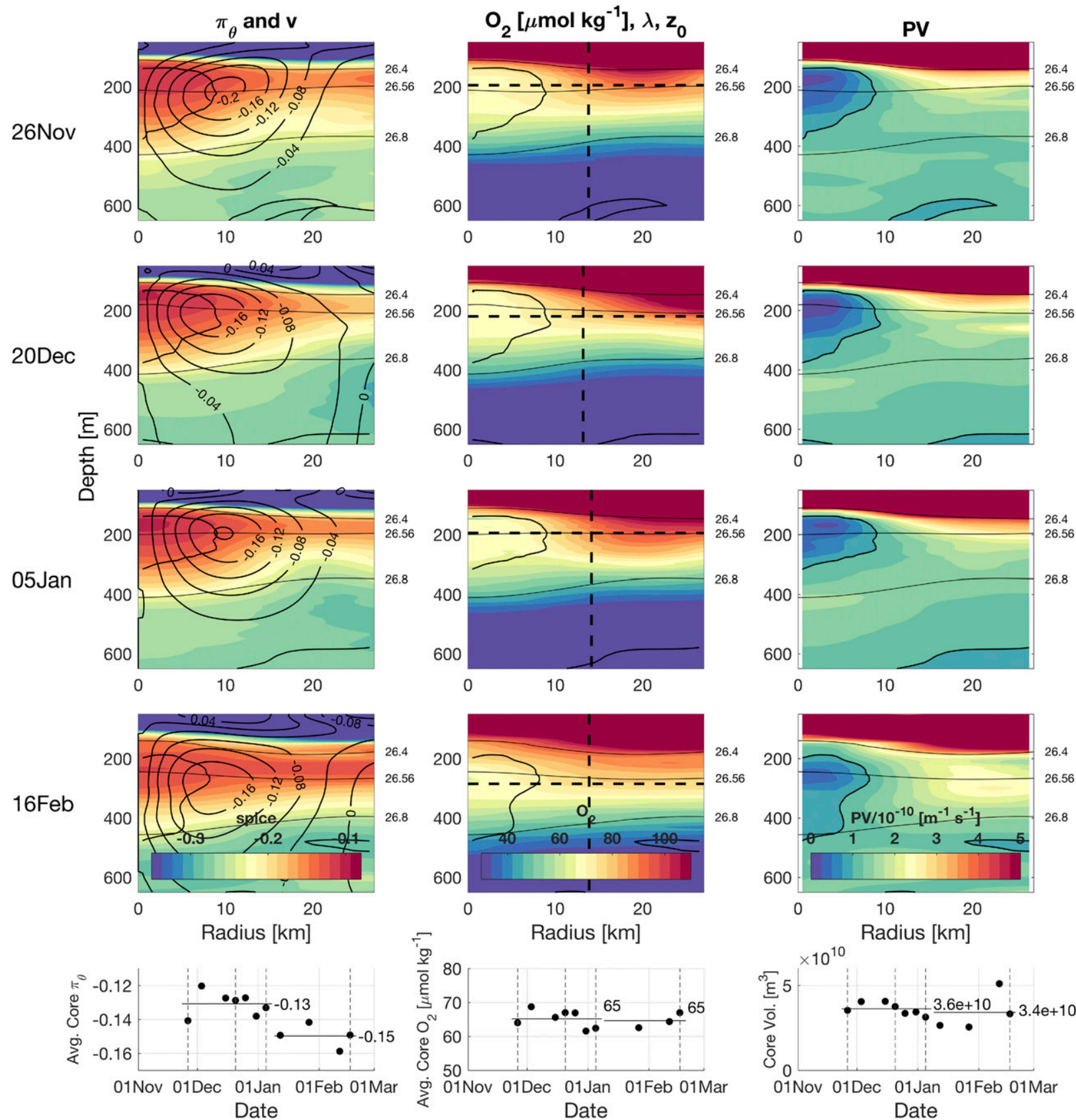

FIG. 12. (left) Interpolated Cuddy spice $\pi_{\theta}$ (colored) and azimuthal velocity $v_{\phi}$ (contours), (center) dissolved $\mathrm{O}_{2}$, and (right) modeled PV (right column) in four of 11 representative analysis windows (first four rows). In the center column, dashed lines indicate core depth and $r=r_{v_{\max }}$. In the center and right column, the solid black contour indicates "core" $\mathrm{PV}=1 \times 10^{-10} \mathrm{~m}^{-1} \mathrm{~s}^{-1}$. Thin black contours in all columns indicate core and bounding isopycnals. The bottom row depicts respective average core property changes in time within the volume bounded by the contoured PV limit. Vertical dashed lines highlight the four selected time windows.

translation, the Cuddy occupied a region of slope with a relatively weak cross-slope topographic gradient, while an increase in slope steepness was coincident with the observed northward acceleration beginning in early January. The degree of connection between this eddy and the seafloor is unknown, since observations did not extend to full depth in this study. The observations do suggest, however, the possibility that eddy azimuthal velocity was nonzero at depths greater than $1000 \mathrm{~m}$. Roughan et al. (2017) discuss the vertical extent of subsurface intensified eddies and relate observations of $T / S$ anomalies many hundreds of meters below an eddy core. 

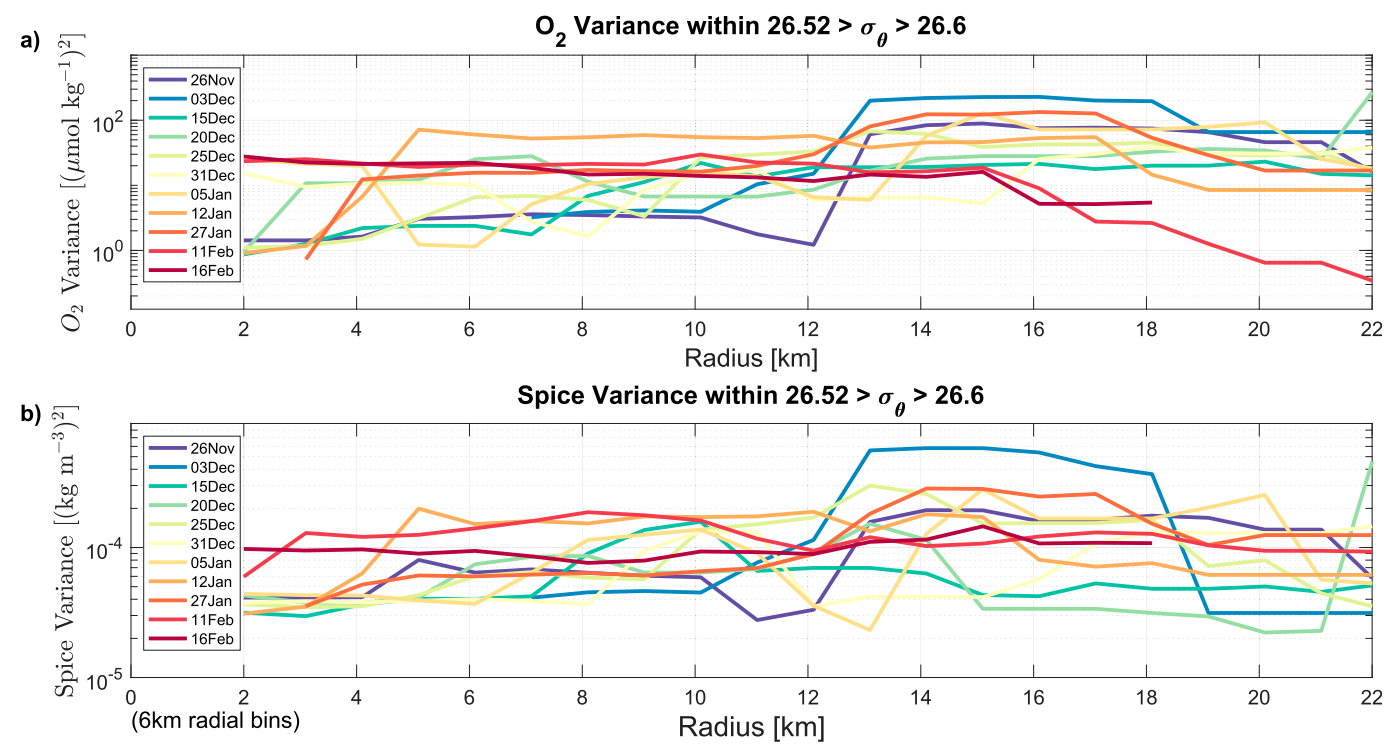

FIG. 13. Average radial profiles of (a) oxygen and (b) spice variance between $\sigma_{\theta}=26.52 \mathrm{~kg} \mathrm{~m}^{-3}$ and $\sigma_{\theta}=$ $26.6 \mathrm{~kg} \mathrm{~m}^{-3}$ for each analysis window. Note $\log y$ axes with different scales.

The alignment of $f /$ water-depth contours, the magnitude of a presumed barotropic mean flow as compared to an expected eddy westward drift speed, and the possibility of Cuddy interaction with the seafloor are in this case consistent with the hypothesis that steady offshore drift was overwhelmed by advection in topographically constrained background flows.

During the period of southwest translation, spanning a significant portion of the month of December and while the Cuddy occupied a flatter region of the slope, background currents were relatively weak and variable (Fig. 6b). It is plausible that at this time translation due to the beta effect may have had a larger influence on the eddy trajectory, in contrast to the stronger northward background flows and translation over steeper sections of the slope observed later in the surveys.

Originally explored through the modeling of eddy formation, adjustment, and evolution (McWilliams and Gent 1986), Burger number selection was observed as the Cuddy traveled over $400 \mathrm{~km}$ in three months. This selection process represents a tendency for a stable equilibrium ratio of vertical to horizontal scales to develop. Values remained in the stable range, increased toward 1, and fell within the range of those observed by Pelland et al. (2013) and cited by D'Asaro (1988b). Both the aspect ratio and ratio of stratification to $f$ changed in a compensating manner, however, resulting in no statistically significant Burger number change. Hebert et al. (1990) similarly studied the evolution of an SCV over the course of two years and observed significant decay along with aspect ratio adjustment. While the ratio of kinetic to available potential energy remained Q (1), total energy decreased fourfold and the Burger number increased in those observations. Here we observed a similar partition of energy as well as decrease in time of potential energy, but a modest and not statistically significant change in Burger number.

Recent repeat observations of the semistationary Lofoten basin eddy made by Seagliders (Yu et al. 2017) reveal the evolution of an eddy slightly larger and stronger than this Cuddy. Comparisons between radial azimuthal velocity profiles, however, show agreement in profile shape with both eddies in cyclogeostrophic balance. Yu et al. (2017) report a core in solid body rotation out to $\sim(1 / 3) r_{v_{\max }}$ and minimum in core vorticity to be $\sim-0.8 f$. Along with a vorticity Rossby number of roughly 0.8 and fluctuating Burger number $\mathscr{O}(1)$, these results are comparable to Cuddy observations and help validate the framework used in studying these submesoscale features. Over the course of their multiyear survey, the authors report strong seasonal changes in background waters and an eddy core that remains largely isolated and persistent.

While the individual Cuddy that was tracked in this study was initially quite isolated (evidenced by a core with low spice and $\mathrm{O}_{2}$ variance), after three months, the eddy geopotential anomaly, Rossby number, potential energy, core spice anomaly, and available salt anomaly all decreased, while core potential vorticity, oxygen variance, instances of fine structure, and eddy vertical scale increased. After the period of increased translation the Cuddy core was also notably deeper. A possible 

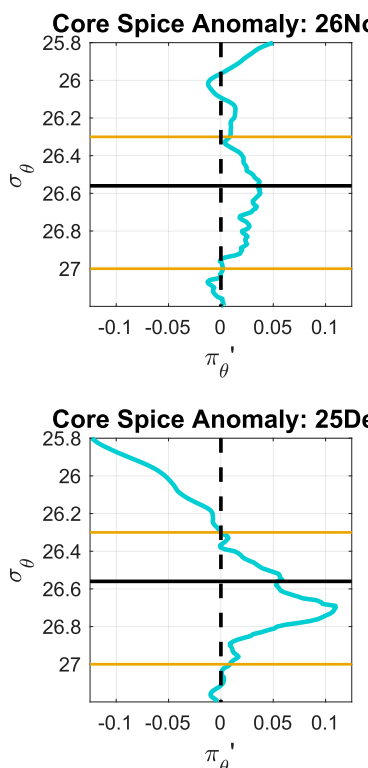

Core Spice Anomaly: 27Jan

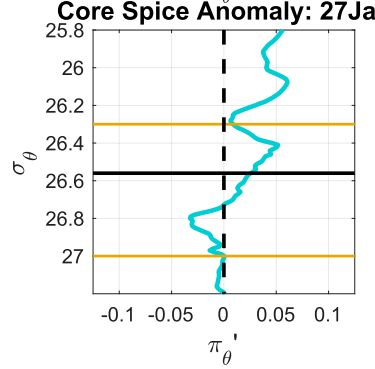

$\times 10^{9}$

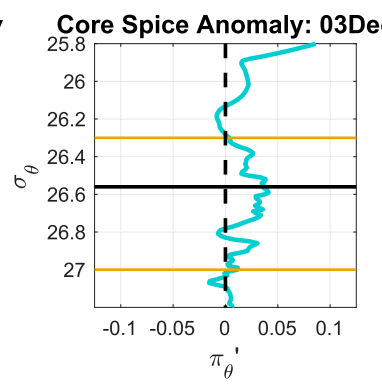

Core Spice Anomaly: 31Dec

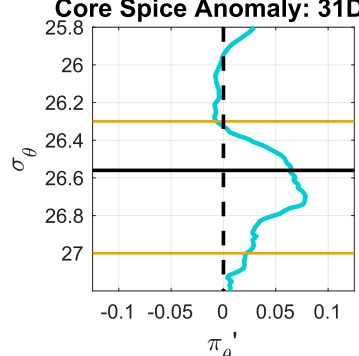

$\pi_{\theta}^{\prime}$

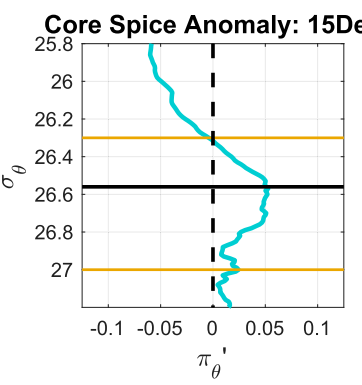

Core Spice Anomaly: 05Jan
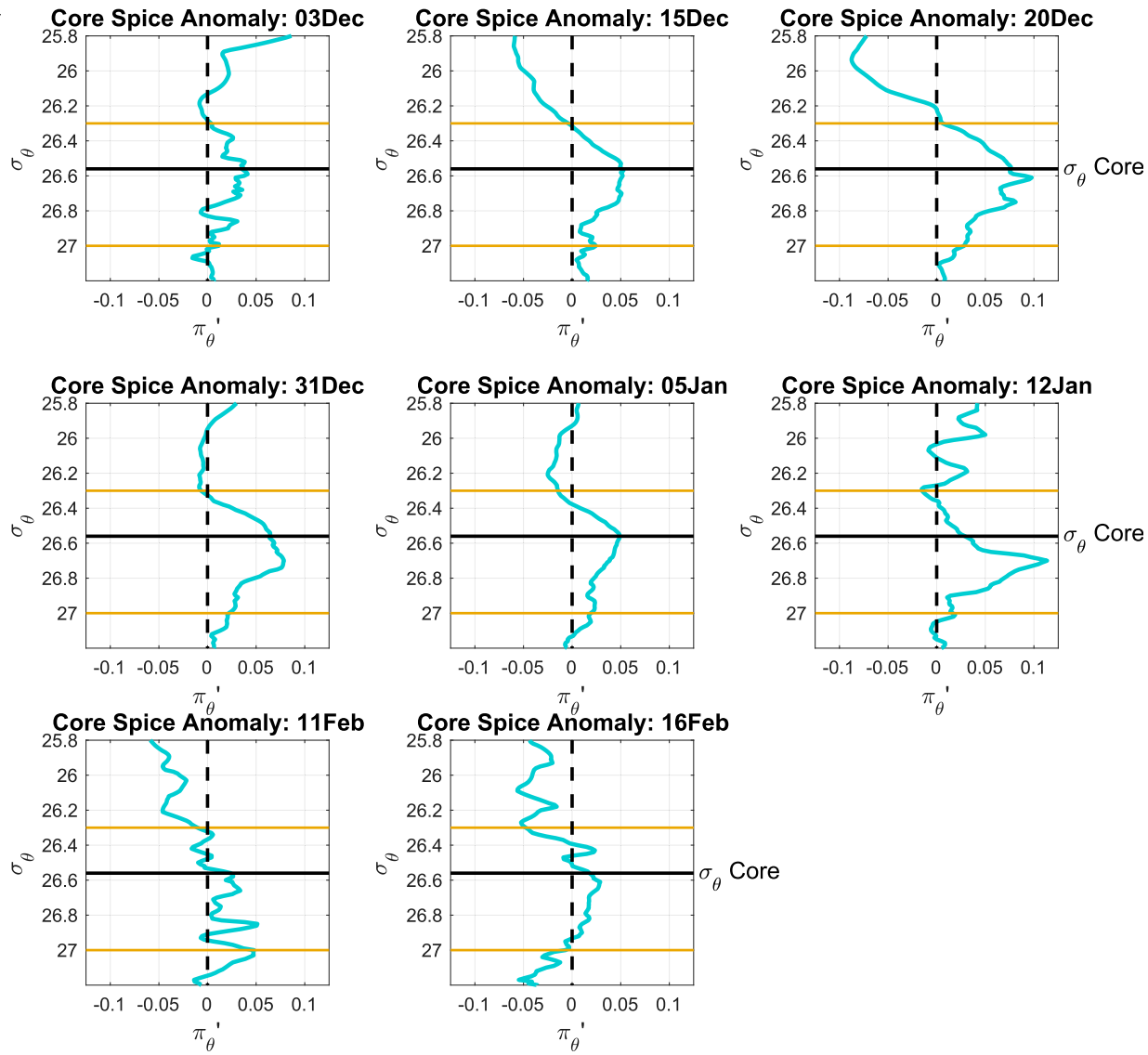

Available Salt Anomaly (relative to mission avg $\mathrm{S}_{\text {ref }}$ )

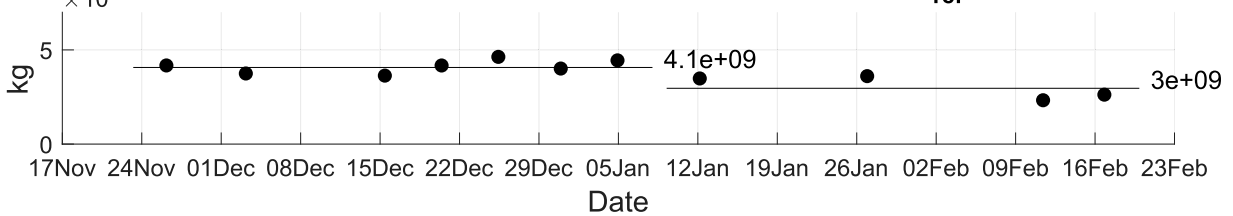

FIG. 14. Spice anomaly profiles within the eddy center for each analysis window. Horizontal lines are density boundaries of the core region (yellow) and the core isopycnal (thick black). The zero spice anomaly reference is in dashed black. The lower figure is available salt anomaly (ASA) within each snapshot vs time, including early and late averages (horizontal lines).

explanation for the loss of total energy and increase in tracer variance is the effect of thermohaline lateral intrusions, which have been implicated in Meddy decay (Armi et al. 1989; Hebert et al. 1990), and which are suggested here by the presence of vertical fine structure. These features at first bounded the Cuddy and were found at radii greater than $r=\lambda$, while later in the survey period, most were found closer to the core. Because individual lateral intrusions could not readily be observed or identified in multiple glider profiles, the horizontal heat and salt fluxes cannot be estimated directly. The relatively rapid eddy evolution observed here, and presumably, correspondingly short lifetimes of any horizontal intrusion features, likely invalidates the use of a steady-state parameterization such as that of Joyce
(1977), as was done in Pelland et al. (2013), for estimating horizontal fluxes of heat and salt due to intrusions in this eddy (Shcherbina et al. 2010).

The presence of vertical fine structure, in addition to the rapid poleward propagation and decrease in the radius at which core waters are trapped, suggests mechanisms by which eddy core water may have been eroded during this study. Erosion of eddy waters, along with movement into different background conditions, are both mechanisms that have the potential to drive eddy evolution. We examined the relative influence of eddy erosion and background change on the bulk properties estimated in this study using an idealized model of a symmetric, geostrophic eddy embedded in uniform stratification. Consider an eddy whose density anomaly takes the form 

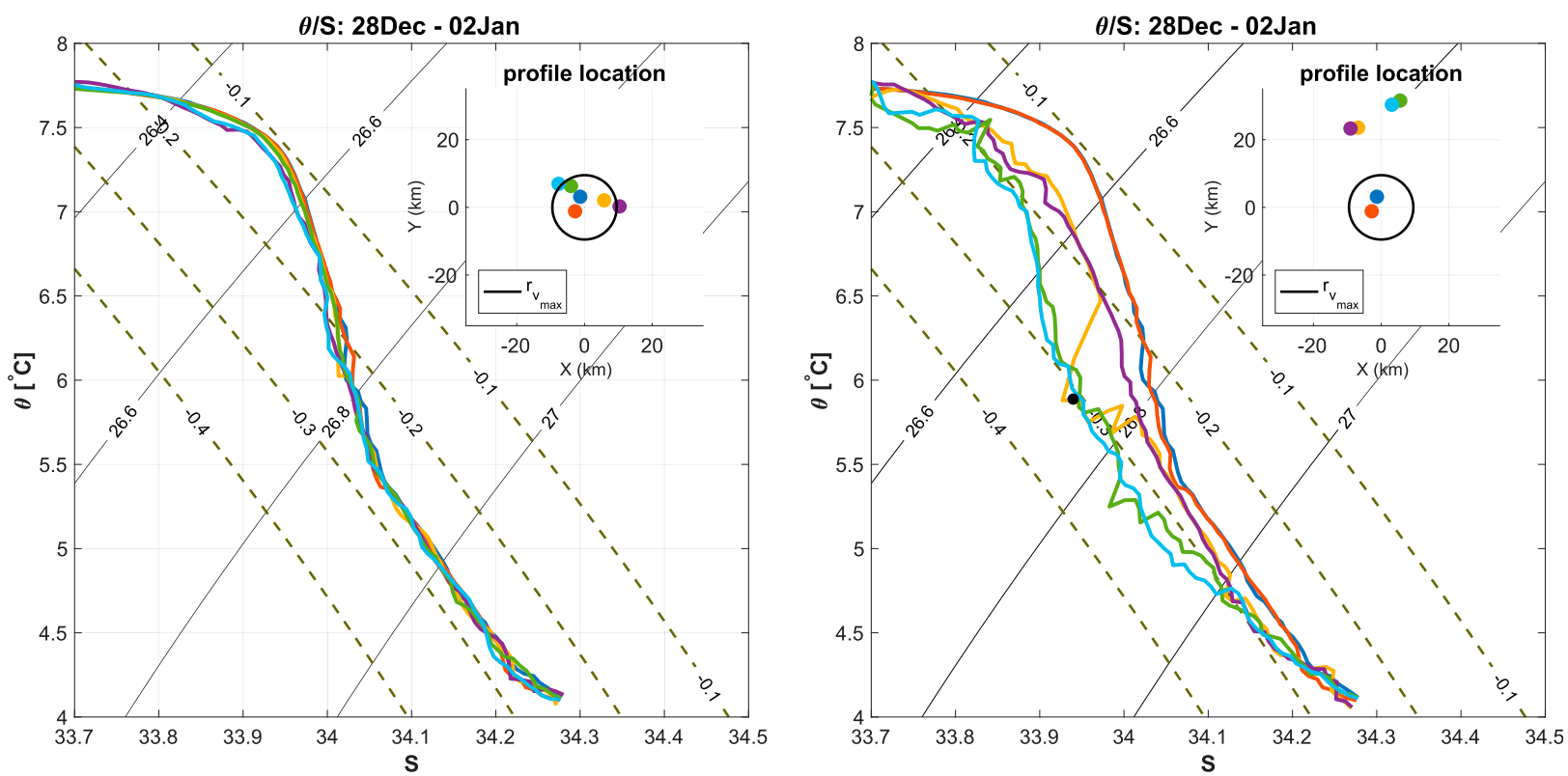

FIG. 15. Two sets of $\theta / S$ profiles, referenced to the Cuddy center position and completed within time window six. Insets detail the midpoint location of each profile relative to the eddy center; black circle indicates the radius of maximum velocity. Profiles are colored according to their location in the inset in each panel with the black dot representing an identified spice curvature extremum.

$$
\rho^{\prime}\left(r, z^{\prime}\right)=\frac{a}{2} \sin \left(\frac{\pi z^{\prime}}{H}\right)\left[\cos \left(\frac{\pi r}{L}\right)+1\right]
$$

where $a, L$, and $H$ are the density anomaly, length scale, and half-height scale respectively and $z^{\prime}=z-z_{0}$ is the vertical coordinate relative to the eddy core depth. Halfheight refers to the eddy half-thickness and is used recognizing the vertical symmetry of the eddy. This form, although simpler than the density anomaly implied by (3), was chosen as an idealization retaining basic Cuddy characteristics in continuous stratification within a finite domain. The density anomaly and velocity field decay to background levels at the domain limits $r=L$ and $z^{\prime}= \pm H$. The exercise consists of examining the change of the three eddy parameters that would be necessary to conserve integrated mass, energy, and PV following a geostrophic adjustment to either background changes, or both erosion and background changes. These two cases reflect Cuddy evolution scenarios that could describe the observations. Solutions are sought for which $a, L$, and $H$ vary to accommodate changes in stratification and latitude while retaining the chosen parametric eddy form specified by (8). The form (8) enables integrals of eddy mass, potential and kinetic energy, and potential vorticity to be expressed analytically as functions of $a, L$, and $H$ over arbitrary cylindrical domains up to $L$ in radius and $2 H$ in height (see appendix B for details). The constraints of mass, total energy, and potential vorticity conservation uniquely determine $a, L$, and $H$ for modest changes in
Coriolis parameter and stratification. Adjustment to erosion of an outer radial annulus or vertical layer can be simulated by seeking final parameters that describe an eddy with reduced amounts of mass, energy, and PV, where reductions are those given by limiting the range of integration of the quantities in the initial eddy over a radius less than $L$ and for a half height less than $H$. Observed ratios of the late to early period eddy properties (maximum geopotential anomaly $\Phi$, height scale $H$, radius scale $L$, Rossby number, Burger number, kinetic, and potential energy) along with $95 \%$ confidence intervals are used in referencing this idealized model to the Cuddy observations (Fig. 17, right). The most robust observed changes include an increase in the vertical scale $H$, a decrease in geopotential amplitude, and a decrease in Rossby number.

Predictions of these ratios in an eddy adjusting to erosion are considered as a function of fractional radial erosion (corresponding to integration over the full original domain). Here, the model results also reflect a $3 \%$ increase in Coriolis parameter and 9\% decrease in background stratification from early to late period observations.

Idealized eddy parameter changes resulting from movement northward into less stratified waters only partially explain observed Cuddy evolution with a decrease in geopotential amplitude and Rossby number. With the inclusion of modeled erosion however, an increase in Burger number and decrease in potential 

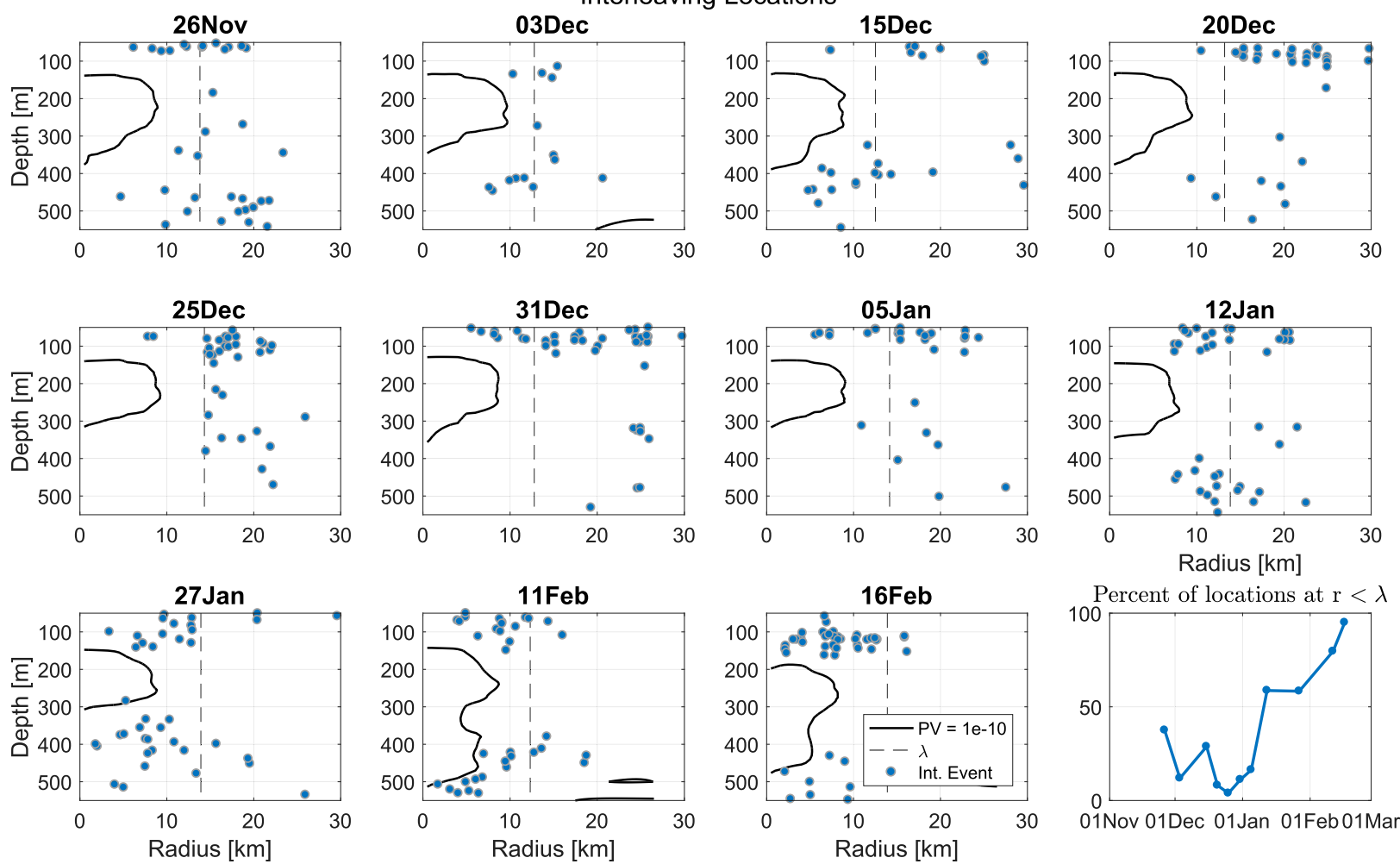

FIG. 16. Locations of fine structure events defined by spice curvature extrema identified within each time window, vs radius and depth. Time advances from left to right by row, and date labels indicate time window midpoints. Scattered points are instances of curvature larger than two standard deviations above the mean of all curvature values found within each time window. The PV contour defined in Fig. 12 is added to identify a core boundary. The radial scale index $r_{\zeta=0}$ is in dashed black. The lower right inset is the percentage of tagged interleaving locations with radii $<r_{\zeta=0}$ vs time.

energy result in better agreement with observed Cuddy changes (Fig. 17). To estimate the influence of erosion (from both horizontal and vertical limits), a cost function was constructed by comparing observed and modeled changes in geopotential anomaly, height scale, length scale, Rossby number, Burger number, kinetic energy, and available potential energy. The sum of the differences between each observed and modeled ratio divided by the corresponding standard deviation of the ratio of late to early observed $t$-distributed parameters was used as a cost function to find the combination of radial and vertical erosion that best matched the observations (appendix B). The results favor a $2.75 \%$ radial erosion alone, combined with the effect of weakened background stratification and movement northward yielding a cost function value of 11.4 (Fig. 17). The corresponding cost function value associated with background changes and no erosion is equal to 11.9. Any inclusion of modeled vertical erosion further increased the cost function to values greater than 11.9. One notable change that the model does not qualitatively predict is a significant increase in the Cuddy vertical scale. This may be partly explained by remembering that the observed vertical scale change is accompanied by large confidence bounds likely affected by the assumption of vertical symmetry about the Cuddy core used in estimating the Cuddy vertical scale (section $4 \mathrm{~b}$ ).

A combination of horizontal erosion and background changes provides the best fit to the observed Cuddy changes, within the estimated confidence bounds on the observed changes. This supports the hypothesis that the observed changes were due to both poleward translation into different waters and core water loss. Confirming the details of the relative losses of dynamical properties of Cuddies and similar subsurface eddies due to exterior erosion likely requires more direct observation of the erosion processes.

\section{Summary and conclusions}

Multiple Seaglider autonomous vehicles observed the evolution of a California Undercurrent eddy over the course of three months of intensive surveying. This feature, exhibiting strong radially symmetric anticyclonic 


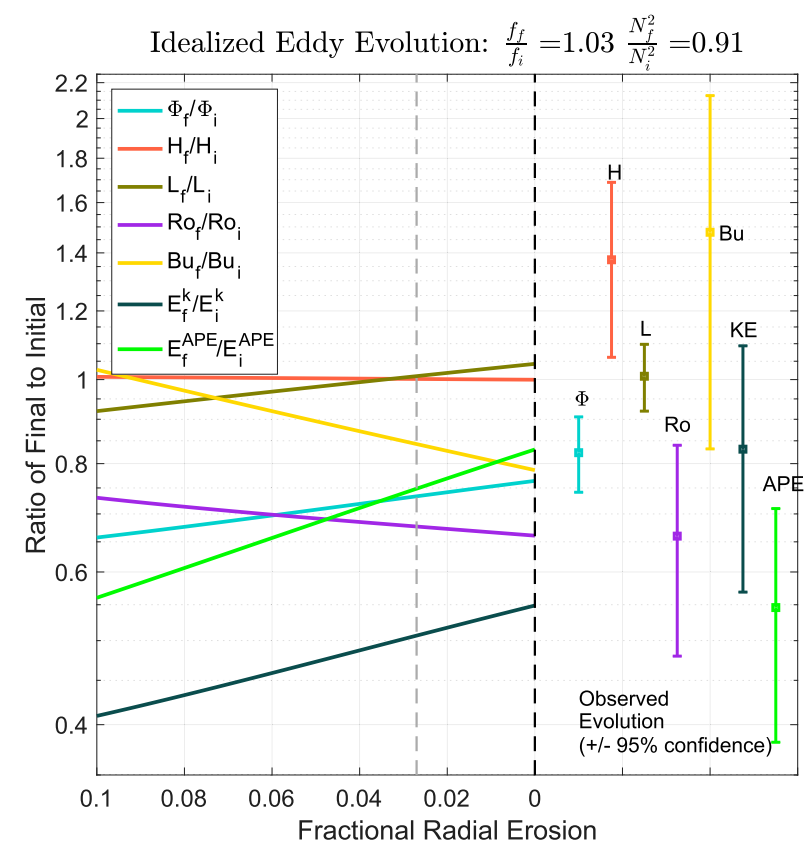

FIG. 17. Idealized geostrophic eddy adjustment to both changes in background conditions and erosion. Changes in the maximum geopotential anomaly $\Phi$, height scale $H$, length scale $L$, Rossby number Ro, Burger number $\mathrm{Bu}$, integrated kinetic energy $E^{k}$, and the eddy component of potential energy $E^{\mathrm{APE}}$ are expressed as ratios of final to initial values. The scattered points with $95 \%$ confidence intervals at the right correspond to observed Cuddy changes and are arranged in order of variable considered (the horizontal position is arbitrary). The black vertical dashed line identifies idealized model adjustments only in response to the prescribed changes in background stratification $(-9 \%)$ and Coriolis parameter $(+3 \%)$. The colored curves to the left of the horizontal axis value of zero are relevant quantity changes in response to modeled erosion as well as the prescribed background changes. Erosion is modeled by integrating conserved quantities horizontally to values less than $L$. This is expressed as a fractional erosion of the original $L$ (i.e., $0.02=2 \%$ erosion of $L_{i}$ ). The gray vertical dashed line identifies the fraction of erosion corresponding to the minimum value of the observation-misfit cost function and equals $2.75 \%$.

rotation and with a core spice greater than inshore undercurrent water levels, had defining characteristics of a submesoscale coherent vortex. With a radius of maximum velocity smaller than the local first baroclinic deformation radius and a Rossby number of order one, the nonlinearity of the vortex described here makes appropriate the label submesoscale (McWilliams 1985; Dewar and Meng 1995; Capet et al. 2008; McWilliams 2016).

Changes in Cuddy scale and kinematics were detectable as it was swept along in poleward flow. These changes were accompanied by the increased prevalence of thermohaline fine structure resulting in increased tracer variance within the Cuddy core region. The migration of these interleaving features, identified using spice curvature, toward the Cuddy core is circumstantially consistent with the erosion of core waters.

Use of an augmented 2D Gaussian model fitted to the Cuddy geopotential anomaly field allowed for the direct computation of velocity, relative vorticity, kinetic energy, and available potential energy. Cuddy evolution was explored by comparing these fields across 11 independent Cuddy snapshots. Between November 2013 and February 2014, Cuddy potential energy decreased while the vertical scale increased. Despite modest decay, the Cuddy retained its bulk shape and structure. Contributing factors to eddy decay were explored using a highly idealized model of a geostrophic eddy and results are consistent, except for changes in the eddy vertical scale, with eddy changes as being due to both erosion of exterior waters and changing background conditions as the eddy moved poleward along the continental slope.

The individual Cuddy tracked in this study did not move significantly offshore during the course of the survey, suggesting that strong alongshore currents carried it, overcoming any dynamic tendency for it to move offshore in the manner observed by Collins et al. (2013). A Cuddy with a larger geopotential anomaly and found in offshore waters would likely be easier to track and monitor, though it remains an open question what fraction of Cuddies reach this mature state. Further in situ observations are needed to resolve the range of behaviors of mature Cuddies and their fate in the energetic nearshore region and beyond. Results of this study indicate that autonomous vehicle surveys are a viable strategy for addressing this need by observing eddy evolution and resolving submesoscale pathways for heat, salt, and energy transport.

Acknowledgments. This work was funded by the National Science Foundation (Grant OCE-1153980). We thank Kirk O'Donnell for his dedication and patience in ensuring that the Seagliders used in this experiment were deployed, well used, and safely recovered. Thanks to Bill Fredericks for his resourcefulness in field and recovery operations. Thanks to Jim Bennett for managing the data stream and organizing/advising on data processing procedures. We are extremely grateful for the crew of the CCGS John P. Tully for assistance in recovering gliders and the USCG Station Grays Harbor for aid in small vessel operations. And many thanks to Tom Young and the crew of the F/V Tommycod for their professionalism and capability. We would additionally like to thank the reviewers of this manuscript for their detailed feedback, suggestions on improving clarity, and help expanding the scope of our interpretation of results. 


\section{APPENDIX A}

\section{Eddy Position Estimates Using the Extended Kalman Smoother}

The eddy center position $[\hat{x}(t), \hat{y}(t)]$ was estimated using the same framework as in eddy tracking: a simplified model was assumed, and glider observations were assimilated into this model using the extended Kalman filter (EKF; Pelland et al. 2018). The simplified model assumed a separable, radially symmetric form of eddy density anomaly

$$
\rho_{\theta}^{\prime}(r, z)=\Delta \rho_{0} F(z) \exp \left(-\frac{r^{2}}{2 R^{2}}\right),
$$

where $\Delta$ is a dimensionless parameter, $\rho_{0}$ is a reference density, $R$ is a horizontal scale, and $F(z)$ is a vertical structure function that was estimated by subtracting an average background profile from Seaglider density data collected from 18 December 2013 to 3 January 2014. The 0-1000-m average eddy geostrophic azimuthal currents due to (A1), $\bar{v}_{g}(r)$, are

$$
\begin{aligned}
\bar{v}_{g}(r) & =\frac{\Delta g r}{f H R^{2}} \exp \left(-\frac{r^{2}}{2 R^{2}}\right) \int_{-H}^{0} \int_{-H}^{z} F(\xi) d \xi d z \\
& =A \frac{r}{R^{2}} \exp \left(-\frac{r^{2}}{2 R^{2}}\right),
\end{aligned}
$$

assuming $v_{g}(r,-H)=0$, where $H=1000 \mathrm{~m}$. Here $A=$ $\Delta g(f H)^{-1} \int_{-H}^{0} \int_{-H}^{z} F(\xi) d \xi d z$, and $g$ and $f$ are as defined in the main text. Movement of the eddy center was assumed to be due to advection by a uniform background flow $\mathbf{u}_{\text {back }}=[U(t), V(t)]$; that is, $d(\hat{x}, \hat{y}) / d t=\mathbf{u}_{\text {back }}$. The assumptions of geostrophic flow that vanishes at $z=-H$ simplified the tracking procedure while retaining the basic characteristics of the system.

The six parameters describing this system $(\hat{x}, \hat{y}, U, V$, $A, R)$ were sorted into a state vector $\mathbf{s}(t)$ whose values were estimated using the EKF. After the completion of each vehicle cycle, the EKF operated by computing the differences between observations from all gliders within a 56-h window and those that would have been expected given the previous best estimate of $\mathbf{s}$, known as the forecast. The estimate of $\mathbf{s}$ was then updated based on these differences multiplied by a gain related to the relative uncertainty between the observations and the forecast. The equations and implementation of the EKF in this study followed the descriptions of Gauthier et al. (1993) and Gelb (1974, their section 6). Estimates of depthaveraged current over each cycle, and isopycnal vertical separation between $\sigma_{\theta}=26.5 \mathrm{~kg} \mathrm{~m}^{-3}$ and $\sigma_{\theta}=26.8 \mathrm{~kg} \mathrm{~m}^{-3}$ in each profile, were used as observations in the EKF.
The EKF, rather than the linear Kalman filter, was used because it can accommodate systems in which the observations are nonlinear functions of the state vector $\mathbf{s}$, as is the case here (Gelb 1974). A forward run of the EKF produces an estimate of $\mathbf{s}(t)$ that reflects observations collected up to $t$; refining this estimate to take into account measurements made after $t$ is known as "smoothing." The smoother is essentially a Kalman filter that runs backward using the final estimate of $\mathbf{s}$ from the forward run as its starting guess. As it runs, it updates the smoothed estimate $\mathbf{s}^{s}$ based on differences between it and the best estimate from the forward run. The smoother was implemented in this study as described in Gelb (1974, their Table 5.2.2). The smoother algorithm provides an estimate of $\mathbf{s}^{s}(t)$ and its error covariance matrix $\mathbf{P}^{s}=E\left(\delta \mathbf{s}^{s} \delta \mathbf{s}^{s \mathrm{~T}}\right)$, where $E(\cdot)$ is the expectation of a random variable, $\delta \mathbf{s}^{s}=\mathbf{s}^{s}-\mathbf{s}^{b}$, and $\mathbf{s}^{b}$ is the true value of the state vector. The $(\hat{x}, \hat{y})$ elements of $\mathbf{s}^{s}$ were used as eddy position estimates to which all observations were referenced, while the elements corresponding to $\mathbf{u}_{\text {back }}$ were used as estimates of the background flow (section 3b). The elements of the covariance matrix corresponding to eddy position were used in the uncertainty calculation in section $4 c$.

\section{APPENDIX B}

\section{Idealized Eddy Adjustment and Evolution}

Consider an idealized axisymmetric geostrophic eddy of the form (8), with sinusoidal structure limited to radius $L$, half height $H$ and density anomaly amplitude $a$. This form, chosen for convenience, is meant to loosely model the Cuddy tracked in this study but with finite radial and vertical extent, in contrast to the form (3). The model eddy is embedded in a background density stratification specified by buoyancy frequency $\overline{N^{2}}$ so that equivalently, the buoyancy frequency within the eddy can be expressed as

$$
N^{2}\left(r, z^{\prime}\right)=\overline{N^{2}}-\widetilde{N^{2}} \cos \left(\frac{\pi z^{\prime}}{H}\right) \cos \left(\frac{\pi r}{2 L}\right),
$$

where $z^{\prime}=z-z_{0}$ is the vertical coordinate relative to the eddy core depth. Eddy buoyancy frequency deficit $\widetilde{N^{2}}$ magnitude is given by $\widetilde{N^{2}}=g a \pi / \rho_{0} H$, where $g$ is gravity, $\rho_{0}$ is reference density, and total density is given by $\rho=\rho_{0}+\rho_{1}\left(z^{\prime}\right)+\rho^{\prime}\left(r, z^{\prime}\right)$. The background density gradient is linear, given by $\rho_{1}=-\overline{N^{2}} \rho_{0} z^{\prime} / g$. With these definition, and the assumption of geostrophic balance throughout, the azimuthal velocity field is given by

$$
v\left(r, z^{\prime}\right)=\frac{-\widetilde{N^{2}} H^{2}}{f L \pi}\left[\cos \left(\frac{\pi z^{\prime}}{H}\right)+1\right] \sin \left(\frac{\pi r}{L}\right) .
$$


Volume integrated mass $M$, kinetic plus potential energy $E$, and potential vorticity $(\mathrm{PV})$ are given by

$$
\begin{aligned}
& M=2 \int_{0}^{Z} \int_{0}^{2 \pi} \int_{0}^{R}\left(\rho_{1}+\rho^{\prime}\right) r d r d \theta d z^{\prime}, \\
& E=2 \int_{0}^{Z} \int_{0}^{2 \pi} \int_{0}^{R}\left\{\left(\frac{1}{2} \rho_{0} v^{2}\right)+\left[g z^{\prime}\left(\rho_{1}+\rho^{\prime}\right)\right]\right\} r d r d \theta d z^{\prime},
\end{aligned}
$$

$$
\mathrm{PV}=2 \int_{0}^{Z} \int_{0}^{2 \pi} \int_{0}^{R}\left[(\zeta+f) \frac{N^{2}}{g}+\frac{1}{\rho_{0}} \frac{\partial v}{\partial z} \frac{\partial \rho}{\partial r}\right] r d r d \theta d z^{\prime},
$$

where $\zeta$ is relative vorticity, the factor two accounts for vertical symmetry, and the integration limits $R$ and $Z$ are limited to $L$ and $H$, respectively, as specified by the forms (8) and (B1). Expressions for integrated mass, energy, and potential vorticity using (B1) and (B2) become

$$
\begin{aligned}
& M=\left.\left.\frac{-\pi \rho_{0} \overline{N^{2}} H^{2} L^{2}}{g}\left(\frac{z^{\prime 2}}{H^{2}}\right)\right|_{0} ^{Z}\left(\frac{r^{2}}{L^{2}}\right)\right|_{0} ^{R}+\left.\left.\frac{2 \rho_{0} \widetilde{N^{2}} H^{2} L^{2}}{g \pi} \frac{-1}{2} \cos \left(\frac{\pi z^{\prime}}{H}\right)\right|_{0} ^{Z}\left[\frac{r^{2}}{L^{2}}+\frac{2 r}{\pi L} \sin \left(\frac{\pi r}{L}\right)+\frac{2}{\pi^{2}} \cos \left(\frac{\pi r}{L}\right)\right]\right|_{0} ^{R} \\
& E^{k}=\left.\frac{3 \rho_{0} \widetilde{N}^{4} H^{5}}{16 \pi f^{2}}\left[\frac{z^{\prime}}{H}+\frac{2}{3 \pi} \sin \left(\frac{\pi z^{\prime}}{H}\right)+\frac{1}{6 \pi} \sin \left(\frac{2 \pi z^{\prime}}{H}\right)\right]\right|_{0} ^{Z} \times\left.\left[\frac{r^{2}}{L^{2}}+\frac{r}{\pi L} \sin \left(\frac{2 \pi r}{L}\right)-\frac{1}{2 \pi^{2}} \cos \left(\frac{2 \pi r}{L}\right)\right]\right|_{0} ^{R} \\
& E^{p}=\left.\left.\frac{-2 \pi \rho_{0} \overline{N^{2}} H^{3} L^{2}}{3}\left(\frac{z^{\prime 3}}{H^{3}}\right)\right|_{0} ^{Z}\left(\frac{r^{2}}{L^{2}}\right)\right|_{0} ^{R}+\left.\frac{\rho_{0} \widetilde{N^{2}} H^{3} L^{2}}{\pi}\left[\frac{1}{\pi} \sin \left(\frac{\pi z^{\prime}}{H}\right)-\frac{z^{\prime}}{H} \cos \left(\frac{\pi z^{\prime}}{H}\right)\right]\right|_{0} ^{Z} \\
& \times\left.\left[\frac{r^{2}}{L^{2}}+\frac{2 r}{\pi L} \sin \left(\frac{\pi r}{L}\right)+\frac{2}{\pi^{2}} \cos \left(\frac{\pi r}{L}\right)\right]\right|_{0} ^{R} \\
& \mathrm{PV}_{1}=\left.\left.\frac{2 \pi f \overline{N^{2}} H L^{2}}{g}\left(\frac{z^{\prime}}{H}\right)\right|_{0} ^{Z}\left(\frac{r^{2}}{L^{2}}\right)\right|_{0} ^{R} \\
& \mathrm{PV}_{2}=\left.\left.\frac{-2 \overline{N^{2}} \widetilde{N^{2}} H^{3}}{f g}\left[\frac{z^{\prime}}{H}+\frac{1}{\pi} \sin \left(\frac{\pi z^{\prime}}{H}\right)\right]\right|_{0} ^{Z}\left[\frac{r}{\pi L} \sin \left(\frac{\pi r}{L}\right)\right]\right|_{0} ^{R} \\
& \mathrm{PV}_{3}=\left.\left.\frac{-f \widetilde{N^{2}} H L^{2}}{g}\left[\sin \left(\frac{\pi z^{\prime}}{H}\right)\right]\right|_{0} ^{Z}\left[\frac{r^{2}}{L^{2}}+\frac{2 r}{\pi L} \sin \left(\frac{\pi r}{L}\right)+\frac{2}{\pi^{2}} \cos \left(\frac{\pi r}{L}\right)\right]\right|_{0} ^{R} \\
& \mathrm{PV}_{4}=\left.\frac{\pi^{2} \widetilde{N^{4}} H^{3}}{4 f g}\left[\frac{z^{\prime}}{H}+\frac{1}{2 \pi} \sin \left(\frac{2 \pi z^{\prime}}{H}\right)+\frac{2}{\pi} \sin \left(\frac{\pi z^{\prime}}{H}\right)\right]\right|_{0} ^{Z}\left[\frac{r^{2}}{L^{2}}+\frac{r}{\pi L} \sin \left(\frac{2 \pi r}{L}\right)+\frac{1}{2 \pi^{2}} \cos \left(\frac{2 \pi r}{L}\right)\right. \\
& \left.+\frac{4 r}{\pi L} \sin \left(\frac{\pi r}{L}\right)+\frac{2}{\pi^{2}} \sin ^{2}\left(\frac{\pi r}{L}\right)\right]\left.\right|_{0} ^{R}, \quad \text { and } \\
& \mathrm{PV}_{5}=\left.\left.\frac{-\pi^{2} \widetilde{N^{4}} H^{3}}{8 f g}\left[\frac{z^{\prime}}{H}-\frac{1}{2 \pi} \sin \left(\frac{2 \pi z^{\prime}}{H}\right)\right]\right|_{0} ^{Z}\left[\frac{r^{2}}{L^{2}}-\frac{r}{\pi L} \sin \left(\frac{2 \pi r}{L}\right)-\frac{1}{2 \pi^{2}} \cos \left(\frac{2 \pi r}{L}\right)\right]\right|_{0} ^{R},
\end{aligned}
$$


where $E=E^{k}+E^{p}$ and $\mathrm{PV}=\sum_{i} \mathrm{PV}_{i}$. The $5 \mathrm{PV}$ equations correspond to each term in the substitution and expansion of B5. Given prescribed initial (subscript $i$ ) values $H_{i}, L_{i}, \widetilde{N_{i}^{2}}$, and upper integration limits $Z \leq H$ and $R \leq L$, (B6), (B7) and (B8), and (B9)-(B13) describe integrated mass, energy, and PV for the initial eddy, which comprise three constraints on the final (subscript $f$ ) state unknowns $H_{f}, L_{f}$, and $\widetilde{N}_{f}^{2}$. For modeling eddy adjustment to background stratification and Coriolis parameter variations alone, the integrals are carried out for $Z=H$ and $R=L$. To model eddy adjustment following erosion, the integrals in the above constraints are evaluated over less than the full extent: $Z \leq H_{i}$ and $R \leq L_{i}$.

We consider the adjustment of an initial eddy in two different cases. The first represents eddy advection to a location with different background stratification and Coriolis parameter. The second represents eddy decay combined with the effect of the first case.

The first case considers the response of the eddy to a reduction in background stratification by $9 \%$ to $\overline{N_{f}^{2}}$ and increase in $f$ by $3 \%$ to $f_{f}$. Final scale and strength parameters are found by integrating (B6)-(B13) over the entire initial eddy extent (i.e., $Z=H=H_{i}$ and $R=L=L_{i}$ in these expressions) to find expressions for initial mass $M_{i}$, energy $E_{i}$, and potential vorticity $\mathrm{PV}_{i}$. These equal the final values of these quantities expressed in terms of final stratification $\widetilde{N_{f}^{2}}$, vertical scale $H_{f}$, and horizontal scale $L_{f}$ by

$$
\begin{aligned}
& M_{i}=\frac{\rho_{0}}{g}\left[-\pi \overline{N_{f}^{2}}+\left(\frac{2}{\pi}-\frac{8}{\pi^{3}}\right) \widetilde{N_{f}^{2}}\right] H_{f}^{2} L_{f}^{2}, \\
& E_{i}=\frac{\rho_{0} 3 \widetilde{N_{f}^{4}} H_{f}^{5}}{16 \pi f_{f}^{2}}+\rho_{0}\left[\frac{-2 \pi}{3} \overline{N_{f}^{2}}+\left(\frac{1}{\pi}-\frac{4}{\pi^{3}}\right) \widetilde{N_{f}^{2}}\right] H_{f}^{3} L_{f}^{2},
\end{aligned}
$$

and

$$
\mathrm{PV}_{i}=\frac{2 \pi f_{f} \overline{N_{f}^{2}} H_{f} L_{f}^{2}}{g}+\frac{\pi^{2} \widetilde{N_{f}^{2}} H_{f}^{3}}{8 f_{f} g} .
$$

This set of three equations in unknowns is easily reduced to a set of two equations in a pair of unknowns that can be solved numerically to obtain $\widetilde{N_{f}^{2}}$, $H_{f}$, and $L_{f}$. Since the eddy horizontal extent greatly exceeds its vertical extent, an approximate version of the system (B14)-(B16) neglecting the first (kinetic energy) term in (B15) and last term in (B16) yields a quadratic system that can be solved analytically. The analytic solutions serve as useful initial guesses to the numerical solution of the full (B14)-(B16). The result is that $H$ is independent of background stratification, $L$ varies as its inverse square root, and there exists only a small range in Coriolis parameter, $\sim f_{i}$ $(1 \pm 0.15)$, within which solutions of the specified form exist.

The second adjustment case considers eddy decay along with changes in the background stratification and $f$. Eddy decay is modeled by evaluating integrals in (B6)-(B13) to $Z$ and $R$, where $Z / H<1$ and $R / L<1$, to form the initial quantities on the left side of (B14)(B16). Changes in the ratios of final to initial values of $\Phi$, $H, L, \mathrm{Ro}, \mathrm{Bu}, E^{k}$, and $E^{\mathrm{APE}}$ are explored as a function of the radial and vertical erosion of the initial eddy, or alternatively, as the decrease in the extent of radial and vertical integration in (B6)-(B13) that form constraints on the final parameters. Only the eddy component of potential energy $E^{\mathrm{APE}}$ is considered and employed below such that comparison can be made to the observed eddy APE.

To find the combination of vertical and horizontal erosion that, when combined with the effect of northward translation into less stratified waters, best explains the observations, a cost function was employed to minimize the differences between the seven observed and model predicted ratios (Fig. 17). Cost is defined as the sum of the deviation of each model ratio from its observed value scaled by the standard deviation of assumed $t$-distributed late to early observed ratios. That is,

$$
\begin{aligned}
\operatorname{cost}= & \frac{\left(\frac{\Phi_{f}}{\Phi_{i}}\right)_{\text {model }}-\left(\frac{\Phi_{\text {late }}}{\Phi_{\text {early }}}\right)_{\text {obs. }}}{\sigma_{\Phi}}+\frac{\left(\frac{H_{f}}{H_{i}}\right)_{\text {model }}-\left(\frac{H_{\text {late }}}{H_{\text {early }}}\right)_{\text {obs. }}}{\sigma_{H}}+\frac{\left(\frac{L_{f}}{L_{i}}\right)_{\text {model }}-\left(\frac{L_{\text {late }}}{L_{\text {early }}}\right)_{\text {obs. }}}{\sigma_{L}}+\frac{\left(\frac{E_{f}^{k}}{E_{i}^{k}}\right)_{\text {model }}-\left(\frac{\mathrm{KE}_{\text {late }}}{\mathrm{Ke}_{\text {early }}}\right)_{\text {obs. }}}{\sigma_{\mathrm{KE}}} \\
& +\frac{\left(\frac{E_{f}^{\mathrm{APE}}}{E_{i}^{\mathrm{APE}}}\right)_{\text {model }}-\left(\frac{\mathrm{APE}_{\text {late }}}{\mathrm{APE}_{\text {early }}}\right)_{\text {obs. }}}{\sigma_{\mathrm{APE}}}+\frac{\left(\frac{\mathrm{Ro}_{f}}{\mathrm{Ro}}\right)_{\text {model }}-\left(\frac{\mathrm{Ro}_{\text {late }}}{\mathrm{Ro}_{\text {early }}}\right)_{\text {obs. }}}{\sigma_{\mathrm{Ro}}}+\frac{\left(\frac{\mathrm{Bu}_{f}}{\mathrm{Bu}_{i}}\right)_{\text {model }}-\left(\frac{\mathrm{Bu}_{\text {late }}}{\mathrm{Bu}_{\text {early }}}\right)_{\text {obs. }} .}{\sigma_{\mathrm{Bu}}}
\end{aligned}
$$


The standard deviation used to scale each cost term is defined assuming that the observed early and late values of each quantity are samples from separate statistical distributions. Using two $t$ distributions for each quantity, one centered on the early mean and the other on the late mean, the standard deviation is computed of the ratio of late to early distributions. Confidence limits are then computed using this distribution of late to early ratios (Fig. 17, right). The total cost was computed for a range of integration limits $Z / H \leq 1$ and $R / L \leq 1$ in (B6)-(B13). This value was then minimized in each case to find the combination of integration limits that best matched the observations. The minimum cost value for case one, reflecting eddy adjustment only to changes in background stratification and Coriolis parameter, is 11.9. In case two, reflecting eddy adjustment in response to changes in background stratification, Coriolis parameter, and erosion, the minimum cost value of 11.4 is associated with no vertical erosion and $2.75 \%$ horizontal erosion (Fig. 17).

\section{REFERENCES}

Armi, L., D. Hebert, N. Oakey, J. F. Price, P. L. Richardson, T. H. Rossby, and B. Ruddick, 1989: Two years in the life of a Mediterranean salt lens. J. Phys. Oceanogr., 19, 354-370, https://doi.org/ 10.1175/1520-0485(1989)019<0354:TYITLO > 2.0.CO;2.

Auad, G., D. Roemmich, and J. Gilson, 2011: The California Current System in relation to the northeast Pacific Ocean circulation. Prog. Oceanogr., 91, 576-592, https://doi.org/ 10.1016/j.pocean.2011.09.004.

Bosse, A., and Coauthors, 2016: Scales and dynamics of Submesoscale Coherent Vortices formed by deep convection in the northwestern Mediterranean Sea.J. Geophys. Res. Oceans, 121, 7716-7742, https://doi.org/10.1002/2016JC012144.

Bretherton, F. P., R. E. Davis, and C. B. Fandry, 1976: A technique for objective analysis and design of oceanographic experiments applied to MODE-73. Deep-Sea Res. Oceanogr. Abstr., 23, 559-582, https://doi.org/10.1016/0011-7471(76)90001-2.

Capet, X., J. C. McWilliams, M. J. Molemaker, and A. F. Shchepetkin, 2008: Mesoscale to submesoscale transition in the California Current System. Part I: Flow structure, eddy flux, and observational tests. J. Phys. Oceanogr., 38, 29-43, https://doi.org/10.1175/2007JPO3671.1.

Chelton, D. B., M. G. Schlax, and R. M. Samelson, 2011: Global observations of nonlinear mesoscale eddies. Prog. Oceanogr., 91, 167-216, https://doi.org/10.1016/j.pocean.2011.01.002.

Chereskin, T. K., P. P. Morris, P. M. Niiler, P. M. Kosro, R. L. Smith, S. R. Ramp, C. A. Collins, and D. L. Musgrave, 2000: Spatial and temporal characteristics of the mesoscale circulation of the California Current from eddy-resolving moored and shipboard measurements. J. Geophys. Res., 105, 12451269, https://doi.org/10.1029/1999JC900252.

Collins, C. A., T. Margolina, T. A. Rago, and L. Ivanov, 2013: Looping RAFOS floats in the California Current System. DeepSea Res. II, 85, 42-61, https://doi.org/10.1016/j.dsr2.2012.07.027.

Connolly, T. P., B. Hickey, I. Shulman, and R. E. Thomson, 2014: Coastal trapped waves, alongshore pressure gradients, and the California Undercurrent. J. Phys. Oceanogr., 44, 319-342, https://doi.org/10.1175/JPO-D-13-095.1.
D'Asaro, E. A., 1988a: Generation of submesoscale vortices: A new mechanism. J. Geophys. Res., 93, 6685-6693, https://doi.org/ 10.1029/JC093iC06p06685.

- 1988b: Observations of small eddies in the Beaufort Sea. J. Geophys. Res., 93, 6669-6684, https://doi.org/10.1029/ JC093iC06p06669.

Dewar, W. K., and H. Meng, 1995: The propagation of submesoscale coherent vortices. J. Phys. Oceanogr., 25, 1745-1770, https:// doi.org/10.1175/1520-0485(1995)025<1745:TPOSCV>2.0.CO;2.

Early, J. J., R. M. Samelson, and D. B. Chelton, 2011: The evolution and propagation of quasigeostrophic ocean eddies. J. Phys. Oceanogr., 41, 1535-1555, https://doi.org/10.1175/2011JPO4601.1.

Elliott, B., and T. Sanford, 1986: The subthermocline lens D1. Part II: Kinematics and dynamics. J. Phys. Oceanogr., 16, 549-561, https://doi.org/10.1175/1520-0485(1986)016<0549: TSLDPI $>2.0 . \mathrm{CO} ; 2$

Eriksen, C. C., 2017a: Physical data collected from Seaglider SG189 during Cuddy Survey in the North Pacific Ocean, Coastal Waters of Washington/Oregon deployed from 2014-01-16 to 2014-04-27 (NCEI Accession 0162344). Version 1.1, NOAA National Centers for Environmental Information, accessed 7 April 2017, https://data.nodc.noaa.gov/cgi-bin/iso?id=gov.noaa.nodc:0162344.

_, 2017b: Physical data collected from Seaglider SG194 during Cuddy Survey in the North Pacific Ocean, Coastal Waters of Washington/Oregon deployed from 2013-10-25 to 2014-05-10 (NCEI Accession 0162349). Version 1.1, NOAA National Centers for Environmental Information, accessed 7 April 2017, https://data.nodc.noaa.gov/cgi-bin/iso?id=gov.noaa.nodc:0162349.

— 2017c: Physical data collected from Seaglider SG195 during Cuddy Survey in the North Pacific Ocean, Coastal Waters of Washington/Oregon deployed from 2013-10-25 to 2014-02-22 (NCEI Accession 0162357). Version 1.1, NOAA National Centers for Environmental Information, accessed 7 April 2017, https://data.nodc.noaa.gov/cgi-bin/iso?id=gov.noaa.nodc:0162357. , T. J. Osse, R. D. Light, T. Wen, T. W. Lehman, P. L. Sabin, J. W. Ballard, and A. M. Chiodi, 2001: Seaglider: A long-range autonomous underwater vehicle for oceanographic research. IEEE J. Oceanic Eng., 26, 424-436, https://doi.org/10.1109/48.972073.

Flament, P., 2002: A state variable for characterizing water masses and their diffusive stability: Spiciness. Prog. Oceanogr., 54, 493-501, https://doi.org/10.1016/S0079-6611(02)00065-4.

Garfield, N., C. A. Collins, R. G. Paquette, and E. Carter, 1999: Lagrangian exploration of the California Undercurrent, 199295. J. Phys. Oceanogr., 29, 560-583, https://doi.org/10.1175/ 1520-0485(1999)029<0560:LEOTCU>2.0.CO;2.

Gauthier, P., P. Courtier, and P. Moll, 1993: Assimilation of Simulated Wind Lidar Data with a Kalman Filter. Mon. Wea. Rev., 121, 1803-1820, https://doi.org/10.1175/1520-0493(1993)121<1803: AOSWLD $>2.0 . \mathrm{CO} ; 2$.

Gelb, A., 1974: Applied Optimal Estimation. MIT Press, 374 pp.

Hebert, D., N. Oakey, and B. Ruddick, 1990: Evolution of a Mediterranean salt lens: Scalar properties. J. Phys. Oceanogr., 20, 1468-1483, https://doi.org/10.1175/1520-0485(1990)020<1468: EOAMSL $>2.0 . \mathrm{CO} ; 2$.

Hickey, B., 1979: The California Current System-Hypotheses and facts. Prog. Oceanogr., 8, 191-279, https://doi.org/10.1016/ 0079-6611(79)90002-8.

Holton, J. R., 1972: An Introduction to Dynamic Meteorology. 3rd ed. Academic Press, 511 pp.

Huyer, A., R. L. Smith, and B. M. Hickey, 1984: Observations of a warm-core eddy off Oregon, January to March 1978. Deep-Sea Res., 31A, 97-117, https://doi.org/10.1016/01980149(84)90018-9. 
_ J. Barth, P. Korso, R. Shearman, and R. Smith, 1998: Upperocean water mass characteristics of the California Current, summer 1993. Deep-Sea Res. II, 45, 1411-1442, https://doi.org/ 10.1016/S0967-0645(98)80002-7.

Ide, K., and M. Ghil, 1998a: Extended Kalman filtering for vortex systems: Part I: Methodology and point vortices. Dyn. Atmos. Oceans, 27, 301-332, https://doi.org/10.1016/S0377-0265(97)00016-X.

$\ldots$, and _ 1998b: Extended Kalman filtering for vortex systems: Part II: Rankine vortices and observing-system design. Dyn. Atmos. Oceans, 27, 333-350, https://doi.org/10.1016/ S0377-0265(97)00017-1.

Joyce, T., 1977: A note on the lateral mixing of water masses. J. Phys. Oceanogr., 7, 626-629, https://doi.org/10.1175/15200485(1977)007<0626:ANOTLM>2.0.CO;2.

Le Traon, P., 1990: A method for optimal analysis of fields with spatially variable mean. J. Geophys. Res., 95, 13 543-13 547, https://doi.org/10.1029/JC095iC08p13543.

Lukas, R., and F. Santiago-Mandujano, 2001: Extreme water mass anomaly observed in the Hawaii ocean time-series. Geophys. Res. Lett., 28, 2931-2934, https://doi.org/10.1029/2001GL013099.

Martin, J., C. M. Lee, C. Eriksen, C. Ladd, and N. Kachel, 2009: Glider observations of kinematics in a Gulf of Alaska eddy. J. Geophys. Res., 114, C01S05, https://doi.org/10.1029/2008JC004842.

McDougall, T. J., 1987: The vertical motion of submesoscale coherent vortices across neutral surfaces. J. Phys. Oceanogr., 17, 2234-2342, https://doi.org/10.1175/1520-0485(1987)017<2334: TVMOSC $>2.0 . \mathrm{CO} ; 2$.

McWilliams, J. C., 1985: Submesoscale, coherent vortices in the ocean. Rev. Geophys., 23, 165-182, https://doi.org/10.1029/ RG023i002p00165.

_- 1988: Vortex generation through balanced adjustment. J. Phys. Oceanogr., 18, 1178-1192, https://doi.org/10.1175/ 1520-0485(1988)018<1178:VGTBA > 2.0.CO;2.

- 2016: Submesoscale currents in the ocean. Proc. Roy. Soc. London, 472A, https://doi.org/10.1098/rspa.2016.0117.

— herent vortices on the $\beta$-plane. Geophys. Astrophys. Fluid Dyn., 35, 235-255, https://doi.org/10.1080/03091928608245894.

,-- , and N. J. Norton, 1986: The evolution of balanced, low-mode vortices on the $\beta$-plane. J. Phys. Oceanogr., 16, 838-855, https:// doi.org/10.1175/1520-0485(1986)016<0838:TEOBLM>2.0.CO;2.

Molemaker, M. J., J. C. McWilliams, and W. K. Dewar, 2015: Submesoscale instability and generation of mesoscale anticyclones near a separation of the California Undercurrent. J. Phys. Oceanogr., 45, 613-629, https://doi.org/10.1175/JPOD-13-0225.1.

Morel, Y., and J. McWilliams, 1997: Evolution of isolated interior vortices in the ocean. J. Phys. Oceanogr., 27, 727-748, https:// doi.org/10.1175/1520-0485(1997)027<0727:EOIIVI>2.0.CO;2.

Pelland, N. A., C. C. Eriksen, and C. M. Lee, 2013: Subthermocline eddies over the Washington continental slope as observed by Seagliders, 2003-09. J. Phys. Oceanogr., 43, 2025-2053, https:// doi.org/10.1175/JPO-D-12-086.1.

, - - and M. F. Cronin, 2016: Seaglider surveys at Ocean Station Papa: Circulation and water mass properties in a meander of the North Pacific Current. J. Geophys. Res. Oceans, 121, 6816-6846, https://doi.org/10.1002/2016JC011920.

— J. S. Bennett, J. M. Steinberg, and C. C. Eriksen, 2018: Automated glider tracking of a California Undercurrent eddy using the extended Kalman filter. J. Atmos. Oceanic Technol., 35, 2241-2264, https://doi.org/10.1175/JTECH-D-18-0126.1.

Roughan, M., S. Keating, A. Schaeffer, P. Cetina Heredia, C. Rocha, D. Griffin, R. Robertson, and I. Suthers, 2017: A tale of two eddies: The biophysical characteristics of two contrasting cyclonic eddies in the East Australian Current System. J. Geophys. Res. Oceans, 122, 2494-2518, https://doi.org/ 10.1002/2016JC012241.

Ruddick, B., and K. Richards, 2003: Oceanic thermohaline intrusions: observations. Prog. Oceanogr., 56, 499-527, https:// doi.org/10.1016/S0079-6611(03)00028-4.

Rudnick, D. L., and S. T. Cole, 2011: On sampling the ocean using underwater gliders. J. Geophys. Res., 116, C08010, https://doi.org/ 10.1029/2010JC006849.

Schultz Tokos, K., and T. Rossby, 1991: Kinematics and dynamics of a Mediterranean salt lens. J. Phys. Oceanogr., 21, 879-892, https:// doi.org/10.1175/1520-0485(1991)021<0879:KADOAM>2.0.CO;2.

Shcherbina, A. Y., M. C. Gregg, M. H. Alford, and R. R. Harcourt, 2009: Characterizing thermohaline intrusions in the North Pacific subtropical frontal zone. J. Phys. Oceanogr., 39, 27352756, https://doi.org/10.1175/2009JPO4190.1.

,,$-- \ldots$, and -2010 : Three-dimensional structure and temporal evolution of submesoscale thermohaline intrusions in the North Pacific subtropical frontal zone. J. Phys. Oceanogr., 40, 1669-1689, https://doi.org/10.1175/2010JPO4373.1.

Simpson, J. J., T. D. Dickey, and C. J. Koblinsky, 1984: An offshore eddy in the California Current System. Part I: Interior dynamics. Prog. Oceanogr., 13, 5-49, https://doi.org/10.1016/ 0079-6611(84)90004-1.

von Storch, H., and F. W. Zwiers, 2003: Statistical Analysis in Climate Research. 2nd ed. Cambridge University Press, 496 pp.

Thomsen, S., T. Kanzow, G. Krahmann, R. J. Greatbatch, M. Dengler, and G. Lavik, 2016: The formation of a subsurface anticyclonic eddy in the Peru-Chile Undercurrent and its impact on the near-coastal salinity, oxygen, and nutrient distributions. J. Geophys. Res. Oceans, 121, 476-501, https://doi.org/10.1002/ 2015JC010878.

Thomson, R. E., and M. V. Krassovski, 2010: Poleward reach of the California Undercurrent extension. J. Geophys. Res., 115, C09027, https://doi.org/10.1029/2010JC006280.

$\longrightarrow$, and - 2015: Remote alongshore winds drive variability of the California Undercurrent off the British Columbia-Washington coast. J. Geophys. Res. Oceans, 120, 8151-8176, https://doi.org/ 10.1002/2015JC011306.

Torres, H. S., and J. Gomez-Valdes, 2017: Erosion of a California Undercurrent eddy by bottom topography. J. Geophys. Res. Oceans, 122, 3715-3735, https://doi.org/10.1002/2016JC011870.

Yu, L.-S., A. Bosse, I. Fer, K. A. Orvik, E. M. Bruvik, I. Hessevik, and K. Kvalsund, 2017: The Lofoten Basin eddy: Three years of evolution as observed by Seagliders. J. Geophys. Res. Oceans, 122, 6814-6834, https://doi.org/10.1002/2017JC012982. 